Submitted to: J. Phys. A: Math. Gen.

\title{
Discrete breathers in a two-dimensional hexagonal Fermi-Pasta-Ulam lattice
}

\author{
Imran A Butt and Jonathan A D Wattis \\ Theoretical Mechanics, School of Mathematical Sciences, University of Nottingham, \\ University Park, Nottingham, NG7 2RD, UK \\ E-mail: imran.butt@maths.nott.ac.uk, jonathan.wattis@nottingham.ac.uk
}

\begin{abstract}
We consider a two-dimensional Fermi-Pasta-Ulam (FPU) lattice with hexagonal symmetry. Using asymptotic methods based on small amplitude ansatz, at third order we obtain a reduction to a cubic nonlinear Schrödinger equation (NLS) for the breather envelope. However, this does not support stable soliton solutions, so we pursue a higher-order analysis yielding a generalised NLS, which includes known stabilising terms. We present numerical results which suggest that long-lived stationary and moving breathers are supported by the lattice. We find breather solutions which move in an arbitrary direction, an ellipticity criterion for the wavenumbers of the carrier wave, asymptotic estimates for the breather energy, and a minimum threshold energy below which breathers cannot be found. This energy threshold is maximised for stationary breathers, and becomes vanishingly small near the boundary of the elliptic domain where breathers attain a maximum speed. Several of the results obtained are similar to those obtained for the square FPU lattice (Butt \& Wattis, $J$ Phys A, 39, $4955,(2006)$ ), though we find that the square and hexagonal lattices exhibit different properties in regard to the generation of harmonics, and the isotropy of the generalised NLS equation.

PACS numbers: 05.45.-a, 05.45.Yv
\end{abstract}

This version: 10 October 2007

\section{Introduction}

Discrete breathers are time-periodic and spatially localised exact solutions of translationally invariant nonlinear lattices. For a brief review of some general properties of breathers in higher-dimensional systems, see our earlier work [2]. In particular, it is known that while some fundamental properties such as the existence of breathers are not affected by lattice dimension (see Flach et al [9] and Mackay and Aubry [14]), other properties are affected profoundly, for instance, the energy properties of breathers (see Flach et al [8], Kastner [12] and Weinstein [19]). In this paper we investigate how the symmetry of the lattice influences the properties of discrete breathers found therein.

In Hamiltonian systems, stationary breathers occur in one-parameter families. For a certain class of Hamiltonian systems, a critical spatial dimension $d_{c}$ exists such that 
for systems with $d \geq d_{c}$, there exists a positive lower bound on the energy of a breather family, and the breather energies do not approach zero even as the amplitude tends to zero. For lattices with dimension $d<d_{c}$, there is no positive lower bound on the energy of breathers. In other words, the energy of a family of breathers goes to zero with amplitude, and breathers of arbitrarily small energy can be found. The critical dimension $d_{c}$ is typically two. A small amplitude expansion yields the NLS reduction to $\mathrm{i} F_{T}+\Delta F+\kappa|F|^{2 \sigma} F=0$ which has a critical dimension of $d_{c}=2 / \sigma$, with blow-up in NLS occurring when $\kappa>0$ and $d>d_{c}$. For typical lattice potentials, $\sigma=1$ again confirming the critical dimension of $d_{c}=2$.

Marin, Eilbeck and Russell have performed extensive numerical investigations of breather dynamics in two-dimensional lattices [15-17]. Their results suggest that moving breather modes exist (or are at least extremely long-lived), and that the lattice exhibits a strong directional preference whereby breathers can only move along symmetries of the lattice, and in no other direction. Such quasi-one-dimensional behaviour is also observed in higher-dimensional lattices.

The work in this paper follows on from our earlier study [2] of breathers in a two-dimensional lattice with square rotational symmetry; hereupon referred to as the "square" lattice. This lattice has $C_{4}$ symmetry, by which we mean that a rotation through any multiple of $2 \pi / 4=\pi / 2$ about an axis perpendicular to the lattice plane through a lattice site maps the lattice onto itself. In [2], using the semi-discrete multiple-scale method (see Remoissenet [18]), we determined an approximate form for (as well as the properties of) small amplitude breathers in a two-dimensional square Fermi-Pasta-Ulam (FPU) lattice [5]. We found a third-order analysis to be inadequate, since the partial differential equation obtained at this order describing the breather envelope exhibits blow-up. To overcome this, we incorporated higher-order effects in the model, thereby obtaining a modified partial differential equation which includes known stabilising terms. From this, we determined regions of parameter space where breather solutions are expected. Numerical simulations supported the results of our analysis, and suggested that, in contrast to the two-component lattices studied by Marin et al [15-17] (that is, with two degrees of freedom at each lattice site), there is no restriction upon the permitted directions of travel within the one-component square FPU lattice. We also found asymptotic estimates for the breather energy which confirmed the existence of a minimum threshold energy, in agreement with the work of Flach et al [8].

In this paper, we consider a hexagonal electrical transmission lattice (HETL). This two-dimensional network possesses $C_{6}$ (or hexagonal) rotational symmetry. That is, rotation through any multiple of the angle $2 \pi / 6=\pi / 3$ about a lattice site maps the lattice onto itself. The HETL is shown in Figure 1, pictured from a point vertically above the plane of the lattice. We note from Figure 1 that geometrically, the HETL is an arrangement of tessellating triangles (not hexagons). Nevertheless, the arrangement in Figure 1 is referred to as "hexagonal" rather than "triangular" since these descriptions refer to its symmetry properties and not to the geometrical shapes which comprise the array (not all authors follow this convention). One might expect the analysis for 


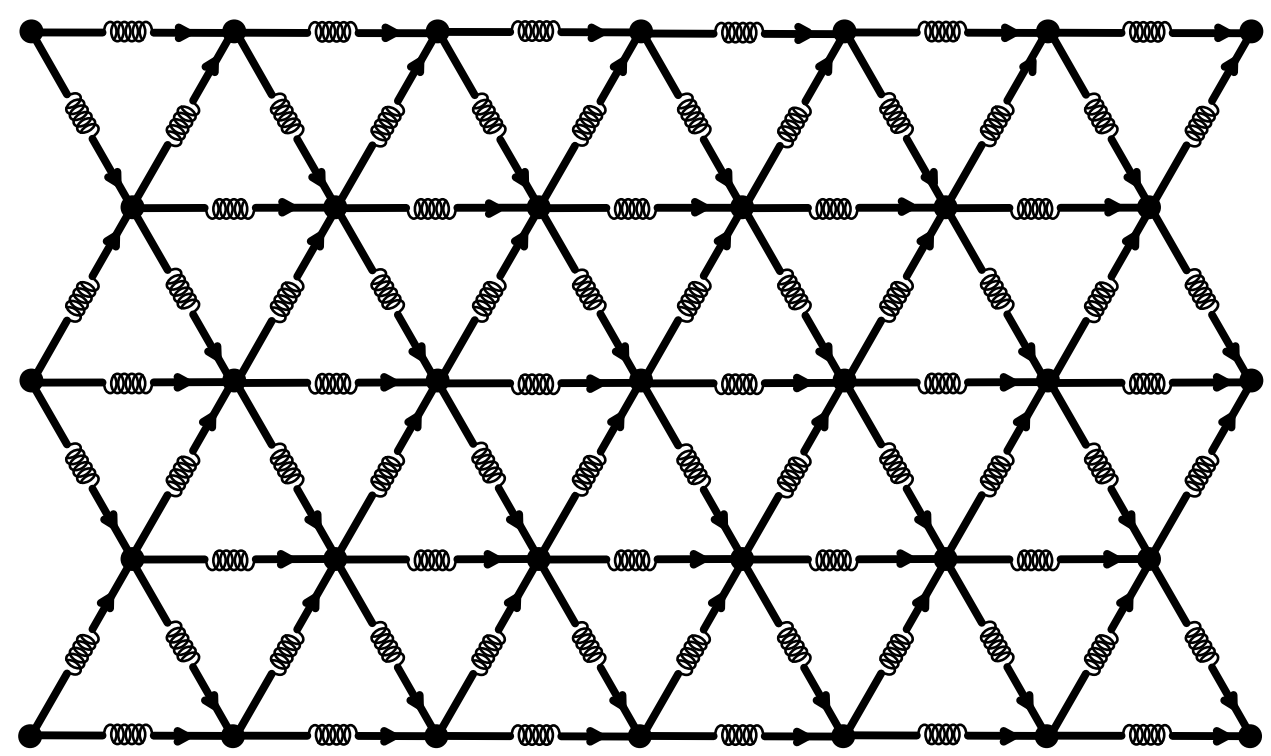

Figure 1: The 2D hexagonal electrical transmission lattice (HETL).

the hexagonal lattice to be more involved, since it is geometrically more complicated in having more links emanating from each node. However, the hexagonal symmetry results in greater isotropy and hence simpler equations than those obtained for the square lattice.

We derive the equations of motion and demonstrate a Hamiltonian formalism in Section 2.2. In Sections 2.6 and 2.7, we present two cases for which the hexagonal FPU lattice equations can be reduced to a two-dimensional nonlinear Schrödinger (NLS) equation with cubic nonlinearity. We consider lattices with a symmetric interaction potential, in which case reduction to a cubic NLS equation can be performed for moving breathers. We find an ellipticity criterion for the wavenumbers of the carrier wave in Section 2.6.1. A reduction to the cubic NLS equation can also be carried out for lattices with an asymmetric potential, provided we consider only stationary breathers.

As expected we find a minimum energy below which breathers cannot exist in the hexagonal FPU lattice. We find that the energy threshold is dependent upon the wavevector of the carrier wave. It is maximised for stationary breathers, and becomes arbitrarily small near the boundary of the elliptic domain.

The cubic NLS equation admits only unstable Townes solitons. Hence, in Section 3, we extend our asymptotic analysis to higher order and find an isotropic generalised NLS equation which incorporates known stabilising terms. Our analytic work is supplemented by numerical simulations presented in Section 4, which suggest that long-lived stationary and moving breather modes are supported by the system. In Section 5, we discuss the results obtained in this paper. 


\section{A two-dimensional hexagonal Fermi-Pasta-Ulam lattice}

\subsection{Preliminaries}

Before we derive the equations governing the HETL, we describe our scheme for indexing the lattice nodes and our choice of basis vectors. We introduce a rectangular lattice with basis vectors $\mathcal{B}=\left\{\mathbf{i}^{\prime}, \mathbf{j}^{\prime}\right\}$, where $\mathbf{i}^{\prime}=\mathbf{i}=[1,0]^{T}$ and $\mathbf{j}^{\prime}=[0, h]^{T}$ (j being $\left.[0,1]^{T}\right)$, illustrated in Figure 2. We use only half of the $(m, n)$ indices, namely, those for which the sum $m+n$ is even. We choose an origin with coordinates $(0,0)$; the position of the site $(m, n)$ is $m \mathbf{i}^{\prime}+n \mathbf{j}^{\prime}$. In order for the hexagonal lattice to be regular, we specify $h=\sqrt{3}$.

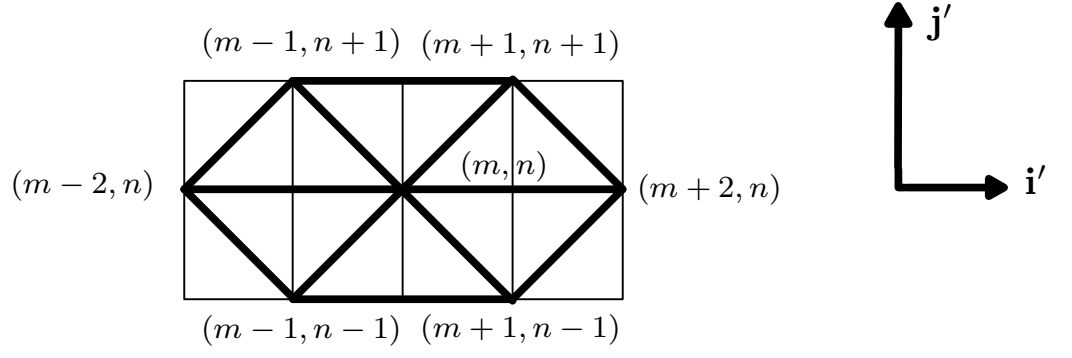

Figure 2: Labelling of nodes in the HETL with basis $\mathcal{B}=\left\{\mathbf{i}^{\prime}, \mathbf{j}^{\prime}\right\}$.

At every node of the HETL lies a nonlinear capacitor (not shown in Figure 1), and between every node and each of its six nearest neighbours is a linear inductor. An enlarged view of the area surrounding the capacitor at $(m, n)$ is shown in Figure 3, where the capacitor is visible. The variable $V_{m, n}$ denotes the voltage across the capacitor $(m, n)$ and $Q_{m, n}$ denotes the total charge stored on the capacitor at $(m, n)$. Also, $I_{m, n}$, $J_{m, n}$ and $K_{m, n}$ are the currents through the inductors immediately to the right of site $(m, n)$ in the directions $\mathbf{e}_{\mathbf{i}}=[2,0]^{T}, \mathbf{e}_{\mathbf{j}}=[1,-\sqrt{3}]^{T}$ and $\mathbf{e}_{\mathbf{k}}=[1, \sqrt{3}]^{T}$ respectively, as illustrated in Figure 3.

\subsection{Derivation of model equations}

To derive the equations relating current, charge and voltage in the lattice we apply Kirchoff's law

$$
\begin{aligned}
& V_{m+2, n}-V_{m, n}=-L \frac{\mathrm{d} I_{m, n}}{\mathrm{~d} t}, \\
& V_{m+1, n-1}-V_{m, n}=-L \frac{\mathrm{d} J_{m, n}}{\mathrm{~d} t}, \\
& V_{m+1, n+1}-V_{m, n}=-L \frac{\mathrm{d} K_{m, n}}{\mathrm{~d} t},
\end{aligned}
$$

where the inductance $L$ is constant. Conservation of charge gives

$$
I_{m-2, n}-I_{m, n}+J_{m-1, n+1}-J_{m, n}+K_{m-1, n-1}-K_{m, n}=\frac{\mathrm{d} Q_{m, n}}{\mathrm{~d} t} .
$$



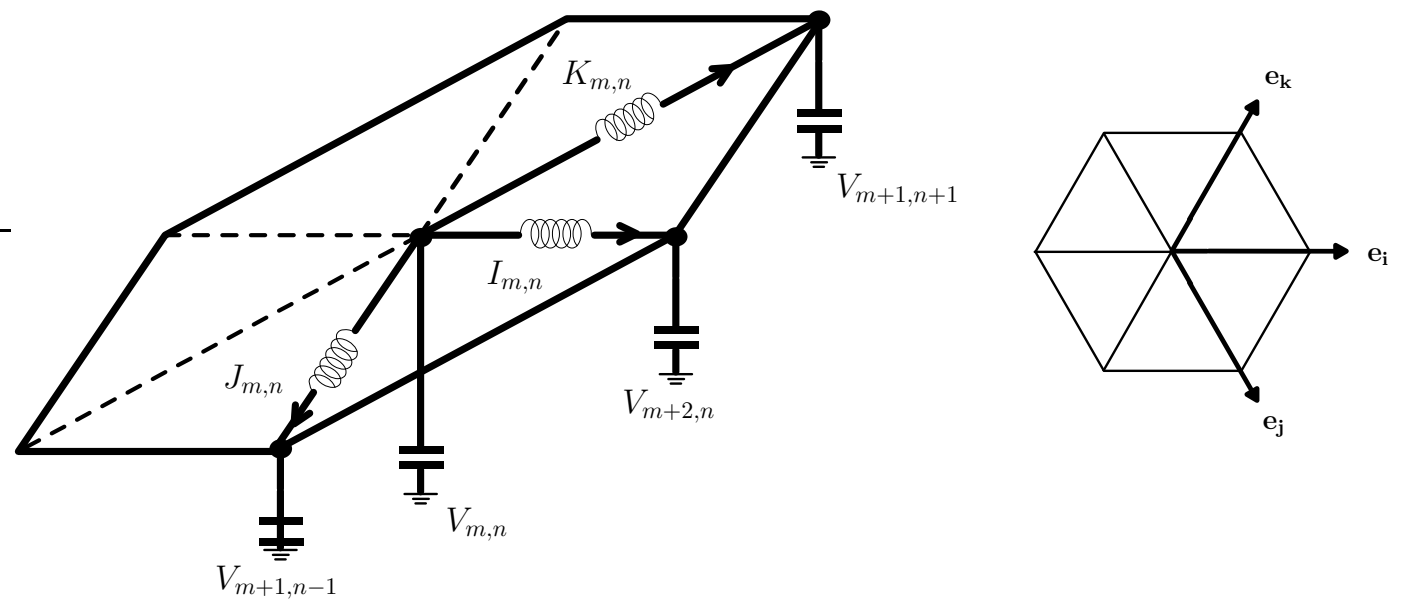

Figure 3: Enlarged view of the HETL at site $(m, n)$.

Differentiating (2.4) with respect to time, and then using (2.1)-(2.3) to find $\dot{I}_{m-2, n}$, $\dot{J}_{m-1, n+1}$ and $\dot{K}_{m-1, n-1}$, we have

$$
\begin{aligned}
L \frac{\mathrm{d}^{2} Q_{m, n}}{\mathrm{~d} t^{2}}= & \left(V_{m+2, n}-2 V_{m, n}+V_{m-2, n}\right)+\left(V_{m+1, n-1}-2 V_{m, n}+V_{m-1, n+1}\right) \\
+ & \left(V_{m+1, n+1}-2 V_{m, n}+V_{m-1, n-1}\right) .
\end{aligned}
$$

Equation (2.5) may be written in the abbreviated form $L \ddot{Q}_{m, n}=\left(\delta_{I}^{2}+\delta_{J}^{2}+\delta_{K}^{2}\right) V_{m, n}$, where the centred second-difference operators are defined by

$$
\begin{aligned}
& \delta_{I}^{2} A_{m, n}=A_{m+2, n}-2 A_{m, n}+A_{m-2, n}, \\
& \delta_{J}^{2} A_{m, n}=A_{m+1, n-1}-2 A_{m, n}+A_{m-1, n+1}, \\
& \delta_{K}^{2} A_{m, n}=A_{m+1, n+1}-2 A_{m, n}+A_{m-1, n-1} .
\end{aligned}
$$

Here, $A_{m, n}$ is an arbitrary quantity referenced by two indices; $\delta_{I}^{2}, \delta_{J}^{2}$ and $\delta_{K}^{2}$ are centred second-difference operators in the directions of $\mathbf{e}_{\mathbf{i}}, \mathbf{e}_{\mathbf{j}}$ and $\mathbf{e}_{\mathbf{k}}$ respectively. Since the voltage $V_{m, n}$ is known in terms of the charge, we reformulate (2.5) in terms of the single quantity $Q_{m, n}$. We invert the capacitor's nonlinear charge-voltage relationship $Q=V C(V)$ (see equations (2.9)-(2.14) of [2] for details), to obtain

$$
V(Q)=\left(Q+a Q^{2}+b Q^{3}+c Q^{4}+d Q^{5}\right) / C_{0},
$$

where $C_{0}=C(0)$. Hence, the HETL equations (2.5) can be written as

$$
\frac{\mathrm{d}^{2} Q_{m, n}}{\mathrm{~d} t^{2}}=\left(\delta_{I}^{2}+\delta_{J}^{2}+\delta_{K}^{2}\right)\left[Q_{m, n}+a Q_{m, n}^{2}+b Q_{m, n}^{3}+c Q_{m, n}^{4}+d Q_{m, n}^{5}\right]
$$

where $m, n \in \mathbb{Z}$ and, by rescaling the time variable, we set $L C_{0}=1$ without loss of generality. Thus we have shown that the equation governing charge in the HETL (2.10) is a two-dimensional analogue of the Fermi-Pasta-Ulam equation

$$
\frac{\mathrm{d}^{2} Q_{j}}{\mathrm{~d} t^{2}}=W^{\prime}\left(Q_{j+1}\right)-2 W^{\prime}\left(Q_{j}\right)+W^{\prime}\left(Q_{j-1}\right)
$$


which is a Hamiltonian system that can be derived from both

$$
H=\sum_{j} \frac{1}{2} \pi_{j}^{2}+W\left(\phi_{j+1}-\phi_{j}\right), \quad \text { and } \quad \widetilde{H}=\sum_{j} \frac{1}{2}\left(P_{j+1}-P_{j}\right)^{2}+W\left(Q_{j}\right),
$$

where $Q_{j}=\phi_{j+1}-\phi_{j}$.

The lattice equations (2.10) can be derived from the Hamiltonian

$$
\begin{gathered}
\widetilde{H}=\sum_{m, n} \frac{1}{2}\left(P_{m+2, n}-P_{m, n}\right)^{2}+\frac{1}{2}\left(P_{m+1, n-1}-P_{m, n}\right)^{2} \\
+\frac{1}{2}\left(P_{m+1, n+1}-P_{m, n}\right)^{2}+\Upsilon\left(Q_{m, n}\right)
\end{gathered}
$$

where $\Upsilon\left(Q_{m, n}\right)$ satisfies $\Upsilon^{\prime}\left(Q_{m, n}\right)=V\left(Q_{m, n}\right)$ given in $(2.9)$, hence

$$
\Upsilon(Q)=\frac{1}{2} Q^{2}+\frac{1}{3} a Q^{3}+\frac{1}{4} b Q^{4}+\frac{1}{5} c Q^{5}+\frac{1}{6} d Q^{6} .
$$

We describe potentials which satisfy $\Upsilon(-Q)=\Upsilon(Q)$ (that is, $a=c=0$ ) as 'symmetric'. The variables $P_{m, n}$ and $Q_{m, n}$ are conjugate momenta and displacement variables of the system; and eliminating $P_{m, n}$ from the equations

$$
\frac{\mathrm{d} Q_{m, n}}{\mathrm{~d} t}=-\left(\delta_{I}^{2}+\delta_{J}^{2}+\delta_{K}^{2}\right) P_{m, n}, \quad \frac{\mathrm{d} P_{m, n}}{\mathrm{~d} t}=-\Upsilon^{\prime}\left(Q_{m, n}\right),
$$

yields (2.10). In Section 2.4, we derive expressions for the energy of breathers given the small amplitude solutions which are obtained in the next section.

\subsection{Asymptotic analysis}

We apply the method of multiple-scales to determine an approximate analytic form for small amplitude breather solutions of (2.10), with slowly varying envelope. We introduce new variables defined by

$$
X=\varepsilon m, \quad Y=\varepsilon h n, \quad \tau=\varepsilon t \quad \text { and } \quad T=\varepsilon^{2} t ;
$$

note the presence of the scaling factor $h$ in the definition of $Y$. We seek solutions of (2.10) of the form

$$
\begin{aligned}
Q_{m, n}(t)= & \varepsilon \mathrm{e}^{\mathrm{i} \psi} F(X, Y, \tau, T)+\varepsilon^{2} G_{0}(X, Y, \tau, T)+\varepsilon^{2} \mathrm{e}^{\mathrm{i} \psi} G_{1}(X, Y, \tau, T)+ \\
& \varepsilon^{2} \mathrm{e}^{2 \mathrm{i} \psi} G_{2}(X, Y, \tau, T)+\varepsilon^{3} H_{0}(X, Y, \tau, T)+\varepsilon^{3} \mathrm{e}^{\mathrm{i} \psi} H_{1}(X, Y, \tau, T)+ \\
& \varepsilon^{3} \mathrm{e}^{2 \mathrm{i} \psi} H_{2}(X, Y, \tau, T)+\varepsilon^{3} \mathrm{e}^{3 \mathrm{i} \psi} H_{3}(X, Y, \tau, T)+\varepsilon^{4} \mathrm{e}^{\mathrm{i} \psi} I_{1}(X, Y, \tau, T)+ \\
& \varepsilon^{4} \mathrm{e}^{2 \mathrm{i} \psi} I_{2}(X, Y, \tau, T)+\varepsilon^{4} \mathrm{e}^{3 \mathrm{i} \psi} I_{3}(X, Y, \tau, T)+\varepsilon^{4} \mathrm{e}^{4 \mathrm{i} \psi} I_{4}(X, Y, \tau, T)+ \\
& \varepsilon^{5} \mathrm{e}^{\mathrm{i} \psi} J_{1}(X, Y, \tau, T)+\cdots+\text { c.c. },
\end{aligned}
$$

where the phase $\psi$ of the carrier wave is given by $k m+l h n+\omega t$ (once again noting the extra factor $h)$, and $\mathbf{k}=[k, l]^{T}$ and $\omega(\mathbf{k})$ are its wavevector and temporal frequency respectively. We substitute the ansatz (2.17) into the lattice equations (2.10) and equate coefficients of each harmonic frequency at each order of $\varepsilon$. After much simplification, this yields the following system of equations:

$\mathcal{O}\left(\varepsilon \mathrm{e}^{\mathrm{i} \psi}\right)$

$$
\omega^{2} F=4 \sin ^{2}(k) F+4 \sin ^{2}\left(\frac{k+l h}{2}\right) F+4 \sin ^{2}\left(\frac{k-l h}{2}\right) F,
$$


$\mathcal{O}\left(\varepsilon^{2} \mathrm{e}^{\mathrm{i} \psi}\right)$

$$
\omega F_{\tau}=2 \sin k[2 \cos k+\cos (l h)] F_{X}+2 h \cos k \sin (l h) F_{Y}
$$

$\mathcal{O}\left(\varepsilon^{2} \mathrm{e}^{2 \mathrm{i} \psi}\right)$

$$
\omega^{2} G_{2}=\left[\sin ^{2}(2 k)+\sin ^{2}(k+l h)+\sin ^{2}(k-l h)\right]\left(G_{2}+a F^{2}\right),
$$

$\mathcal{O}\left(\varepsilon^{3} \mathrm{e}^{\mathrm{i} \psi}\right)$

$$
\begin{aligned}
2 \mathrm{i} \omega F_{T}+F_{\tau \tau}= & {[4 \cos (2 k)+2 \cos k \cos (l h)] F_{X X}+2 h^{2} \cos k \cos (l h) F_{Y Y} } \\
& -4 h \sin k \sin (l h) F_{X Y} \\
& -8 a\left[\sin ^{2}(k)+\sin ^{2}\left(\frac{k+l h}{2}\right)+\sin ^{2}\left(\frac{k-l h}{2}\right)\right]\left[F\left(G_{0}+\bar{G}_{0}\right)+\bar{F} G_{2}\right] \\
& -12 b\left[\sin ^{2}(k)+\sin ^{2}\left(\frac{k+l h}{2}\right)+\sin ^{2}\left(\frac{k-l h}{2}\right)\right]|F|^{2} F
\end{aligned}
$$

$\mathcal{O}\left(\varepsilon^{3} \mathrm{e}^{3 \mathrm{i} \psi}\right)$

$9 \omega^{2} H_{3}=4\left[\sin ^{2}(3 k)+\sin ^{2}\left(\frac{3 k+3 l h}{2}\right)+\sin ^{2}\left(\frac{3 k-3 l h}{2}\right)\right]\left(H_{3}+2 a F G_{2}+b F^{3}\right)$

$\mathcal{O}\left(\varepsilon^{4} e^{0}\right)$

$$
G_{0 \tau \tau}=6 G_{0 X X}+2 h^{2} G_{0 Y Y}+a\left[6\left(|F|^{2}\right)_{X X}+2 h^{2}\left(|F|^{2}\right)_{Y Y}\right] .
$$

Though each equation plays a similar role to its counterpart in the square lattice, equations (2.18)-(2.23) are more complicated. Equation (2.18) is the dispersion relation for the system (2.10). Since we are interested only in solutions for which $F \neq 0,(2.18)$ yields

$$
\omega^{2}=4 \sin ^{2}(k)+4 \sin ^{2}\left(\frac{k+l h}{2}\right)+4 \sin ^{2}\left(\frac{k-l h}{2}\right),
$$

which does not simplify significantly. From equation (2.19), we determine the velocity of the travelling wave $F$, finding

$$
F(X, Y, \tau, T) \equiv F(Z, W, T),
$$

where $Z=X-u \tau$ and $W=Y-v \tau$, and the horizontal and vertical velocity components $(u$ and $v)$ are found to be

$$
u=\frac{-2 \sin (k)[2 \cos (k)+\cos (l h)]}{\omega} \text { and } v=\frac{-2 h \cos (k) \sin (l h)}{\omega} .
$$

Equation (2.26), along with (2.24) enables the elimination of terms involving $G_{1}$ from (2.21) which are not shown. We denote the angle at which the envelope $F$ propagates through the lattice by $\Psi$, which is measured from the direction of the basis vector $\mathbf{e}_{\mathbf{i}}$ to the line of travel and hence is given by $\tan ^{-1}(v / u)$, which in turn depends upon the wavevector k. For both the cases that we consider (namely, symmetric and asymmetric interaction potentials), we find constraints upon the wavenumbers $k$ and $l$ which affect the velocity components $u$ and $v$. By taking $k=\pi / 3$ and $l=\pi / h$ we find $u=v=0$, which corresponds to a static breather; and by choosing $k, l$ values which circle this 
point, we find breathers which can propagate in any direction $(0 \leq \Psi<2 \pi)$; in other words, our analysis suggests that there is no restriction upon the direction of travel through the lattice.

Our aim is to reduce (2.21) to a nonlinear Schrödinger (NLS) equation for $F$. Before this can be done, the quantities $G_{0}$ and $G_{2}$ in (2.21) must be found in terms of $F$. As for the square lattice, it is straightforward to determine $G_{2}$ from the algebraic equation (2.20). However, the partial differential equation (2.23) for $G_{0}$ can be solved for two special cases only; namely, symmetric potentials, in which case the reduction of (2.21) to an NLS equation can be completed even for moving breathers, and also asymmetric potentials, provided we confine our attention to stationary breathers. These two cases are considered in Sections 2.6 and 2.7 respectively, where we also use our breather formulae to generate estimates for the breather energy.

\subsection{Asymptotic estimates for breather energy}

The HETL is a lossless network, meaning that the total electrical energy is conserved. This quantity is related to the Hamiltonian (2.13) by $E=\widetilde{H} / C_{0}$ and so is given by

$$
E=\sum_{m, n} e_{m, n}=\sum_{m, n} \frac{\Upsilon\left(Q_{m, n}\right)}{C_{0}}+\frac{1}{2} L\left(I_{m, n}^{2}+J_{m, n}^{2}+K_{m, n}^{2}\right) .
$$

The electrical energy $e_{m, n}$ associated with a unit of the lattice is (see Figure 3)

$$
e_{m, n}=\frac{\Upsilon\left(Q_{m, n}\right)}{C_{0}}+\frac{1}{2} L\left(I_{m, n}^{2}+J_{m, n}^{2}+K_{m, n}^{2}\right) .
$$

To derive a leading-order estimate for the electrical energy, defined by

$$
E_{0}=\sum_{m, n} e_{m, n}^{(0)}=\sum_{m, n} \frac{Q_{m, n}^{2}}{2 C_{0}}+\frac{L}{2}\left(I_{m, n}^{2}+J_{m, n}^{2}+K_{m, n}^{2}\right),
$$

we use leading-order expressions for each of the terms in the terms in the summand of (2.27). The first term is $Q_{m, n}^{2} /\left(2 C_{0}\right)$; from (2.9), it follows that $V_{m, n} \sim Q_{m, n} / C_{0}$, and so to leading order, (2.27) agrees with the linear approximation to the energy in the capacitor being $Q V / 2$. To find leading-order expressions for the currents $I_{m, n}, J_{m, n}$ and $K_{m, n}$, we use equations $(2.1)-(2.3)$, which imply

$$
\begin{aligned}
& Q_{m+2, n}-Q_{m, n}=-\frac{\mathrm{d} I_{m, n}}{\mathrm{~d} t}, \\
& Q_{m+1, n-1}-Q_{m, n}=-\frac{\mathrm{d} J_{m, n}}{\mathrm{~d} t}, \\
& Q_{m+1, n+1}-Q_{m, n}=-\frac{\mathrm{d} K_{m, n}}{\mathrm{~d} t},
\end{aligned}
$$

where $L C_{0}=1$. The currents are determined by substituting the expression for the breather $Q_{m, n}$ into (2.30)-(2.32) and then integrating with respect to time. We obtain leading-order estimates for the energy of moving breathers in systems with symmetric potentials Section 2.6.2, and in Section 2.7.1, the energies of stationary breathers with asymmetric potentials. 


\subsection{The dispersion relation for the $H E T L$}

In this section, we analyse the dispersion relation (2.24) for the system (2.10). A contour plot of $\omega$ against $k$ and $l$ is shown in Figure 4. Since $w$ is periodic in both $k$ and $l$, with period $2 \pi$ along the $k$-direction, and $2 \pi / h$ in the $l$-direction, we consider only $k$ and $l$ such that $(k, l) \in \mathcal{T}^{2}=[0,2 \pi] \times[0,2 \pi / h]$.

The function $\omega$ is minimised, and assumes the value zero, at the centre of the circular patterns in Figure 4. The minima are located at $(0,0),(2 \pi, 0),(2 \pi, 2 \pi / h)$, $(0,2 \pi / h)$ and $(\pi, \pi / h)$. Plus signs ('+') mark the points in $(k, l)$-space at which $\omega$ is maximised and takes the value $\omega=3$. The maxima lie at the centres of the equilateral triangles, the wavevectors corresponding to these points are denoted $\mathbf{k}_{\mathbf{1}}, \ldots, \mathbf{k}_{\mathbf{6}}$, where

$$
\begin{array}{lll}
\mathbf{k}_{\mathbf{1}}=[\pi / 3, \pi / h]^{T}, & \mathbf{k}_{\mathbf{2}}=[2 \pi / 3,0]^{T}, & \mathbf{k}_{\mathbf{3}}=[4 \pi / 3,0]^{T}, \\
\mathbf{k}_{\mathbf{4}}=[5 \pi / 3, \pi / h]^{T}, & \mathbf{k}_{\mathbf{5}}=[4 \pi / 3,2 \pi / h]^{T}, & \mathbf{k}_{\mathbf{6}}=[2 \pi / 3,2 \pi / h]^{T} .
\end{array}
$$

The arrangement of the points corresponding to these wavevectors in $(k, l)$-space reflects the hexagonal symmetry properties of the lattice (2.10). It may be verified using (2.26) that the velocity components $u$ and $v$ are both zero for each of the wavevectors $\left\{\mathbf{k}_{1}, \ldots, \mathbf{k}_{6}\right\}$.

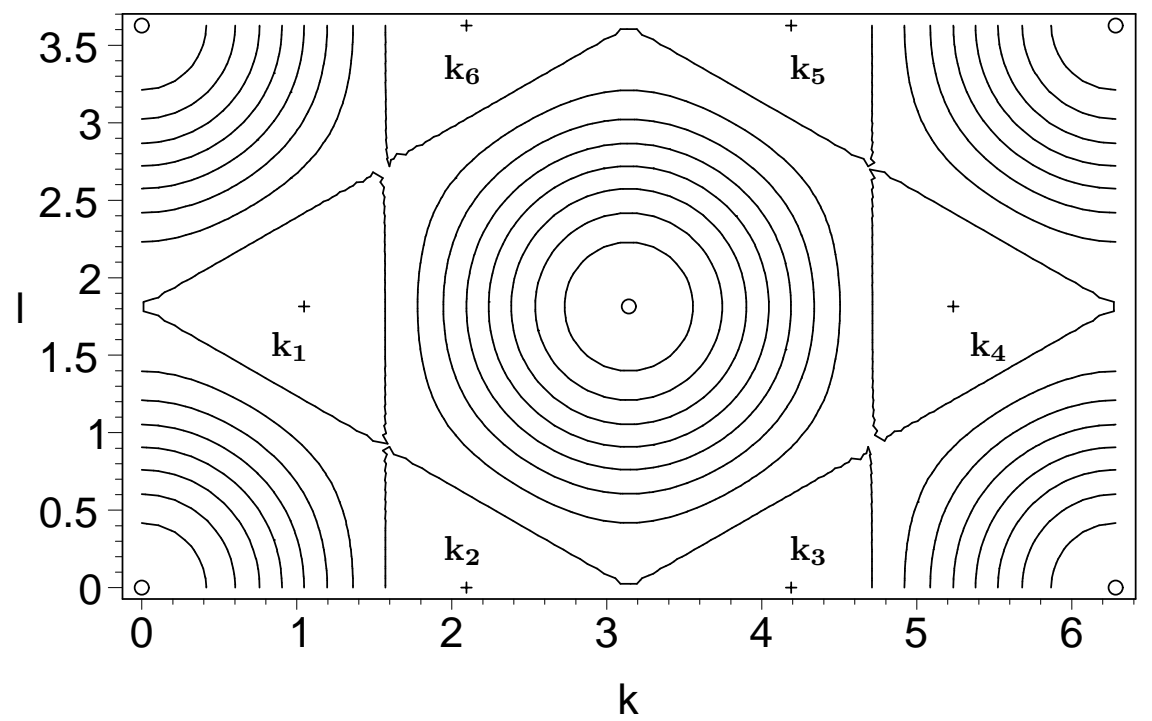

Figure 4: Contour plot of $\omega(\mathbf{k}) ; \omega$ attains its maximum value of 3 at the points $\mathbf{k}_{\mathbf{1}}, \ldots, \mathbf{k}_{\mathbf{6}}$, and is minimised at the points marked ' $\mathrm{o}$ ', where $\omega=0$.

\subsection{Lattices with a symmetric potential}

In this section, we consider lattices with a symmetric interaction potential, where $\Upsilon(Q)$ is even and $\Upsilon^{\prime}(Q)$ has odd symmetry. This corresponds to $a=c=0$ in (2.10). Since there are no even harmonics for vibrations controlled by symmetric potentials, it follows that $G_{0}$ and $G_{2}$ are both zero. In (2.21), the term $F_{\tau \tau}$ is eliminated using $F_{\tau \tau}=u^{2} F_{Z Z}+2 u v F_{Z W}+v^{2} F_{W W}$ which is derived from (2.25). This leads to (2.21) 
being reduced to an NLS equation

$$
\begin{gathered}
2 \mathrm{i} \omega F_{T}+\left[u^{2}-4 \cos (2 k)-2 \cos k \cos (l h)\right] F_{Z Z}+\left[v^{2}-2 h^{2} \cos k \cos (l h)\right] F_{W W} \\
+[2 u v+4 h \sin k \sin (l h)] F_{Z W}+3 b \omega^{2}|F|^{2} F=0,
\end{gathered}
$$

where the velocities $u$ and $v$ are given by (2.26). By applying an appropriate change of variables, we remove the mixed derivative term from (2.34), and reduce the equation to a standard form. To simplify the appearance of subsequent expressions, we denote the coefficients of $F_{Z Z}, F_{W W}$ and $F_{Z W}$ by $D_{1}=u^{2}-4 \cos (2 k)-2 \cos k \cos (l h)$, $D_{2}=v^{2}-2 h^{2} \cos k \cos (l h)$ and $D_{3}=2 u v+4 h \sin k \sin (l h)$ respectively. A suitable transformation is thus

$$
\xi=\frac{h Z}{\sqrt{D_{1}}} \quad \text { and } \quad \eta=\frac{h\left(2 D_{1} W-D_{3} Z\right)}{\sqrt{D_{1}\left(4 D_{1} D_{2}-D_{3}^{2}\right)}},
$$

which implies (2.34) becomes

$$
2 \mathrm{i} \omega F_{T}+3 \nabla^{2} F+3 b \omega^{2}|F|^{2} F=0,
$$

where the differential operator $\nabla^{2} F \equiv F_{\xi \xi}+F_{\eta \eta}$ is isotropic in the $(\xi, \eta)$ variables. An approximation to the Townes soliton solution of (2.36) is given by equation (A6) in the appendix. Substituting the resulting expression for $F$ into (2.17) yields a leading-order expression for the breather

$$
Q_{m, n}(t)=2 \varepsilon \alpha \cos \left[k m+l h n+\left(\omega+\varepsilon^{2} \lambda\right) t\right] \operatorname{sech}(\beta r)+\mathcal{O}\left(\varepsilon^{3}\right),
$$

where $\alpha$ and $\beta$ are determined as described by equation (A5) in the appendix, with $D=3 / 2 \omega, B=3 b \omega / 2$. Further, $r=\sqrt{\xi^{2}+\eta^{2}}$ is found in terms of the physical discrete variables $m$ and $n$ by inverting the transformations (2.35) and reverting back to the variables $Z$ and $W$, using

$$
r^{2}=\xi^{2}+\eta^{2}=\frac{4 h^{2} \varepsilon^{2}\left(D_{2}(m-u t)^{2}+D_{1}(h n-v t)^{2}-D_{3}(m-u t)(h n-v t)\right)}{4 D_{1} D_{2}-D_{3}^{2}} .
$$

The terms $D_{1}, D_{2}$ and $D_{3}$ are known from (2.34), the velocities $u$ and $v$ are given by (2.26) and $\omega$ is given in (2.24).

2.6.1. Determining the domain of ellipticity. We confine our attention to elliptic NLS equations. We seek to determine the region $\mathcal{D}$ of $(k, l)$-parameter space (that is, the twotorus $\left.\mathcal{T}^{2}=[(0,2 \pi)] \times[0,2 \pi / h]\right)$ where the NLS equation (2.34) is elliptic. By definition, this equation is elliptic when $D_{3}^{2}<4 D_{1} D_{2}$, where $D_{1}, D_{2}$ and $D_{3}$ are given in (2.34). Whilst the region $\mathcal{D}$ cannot be specified explicitly, it is simple to find numerically, and is illustrated in Figure 5. Defining the function $e(k, l)=4 D_{1}(k, l) \cdot D_{2}(k, l)-D_{3}(k, l)^{2}$, we are concerned with the region where $e(k, l)>0$. The subdomains have been labelled $\left\{\mathcal{D}_{1}, \ldots, \mathcal{D}_{6}\right\}$ in Figure 5 , where $\mathcal{D}=\mathcal{D}_{1} \cup \ldots \cup \mathcal{D}_{6}$.

Again, the hexagonal symmetry properties of the HETL are reflected clearly in the function $e(k, l)$, which has six maxima (at which $e(k, l)=36$ ), each lying at the centre of one of the closed curves in Figure 5. The maxima of $e(k, l)$ coincide with the six 


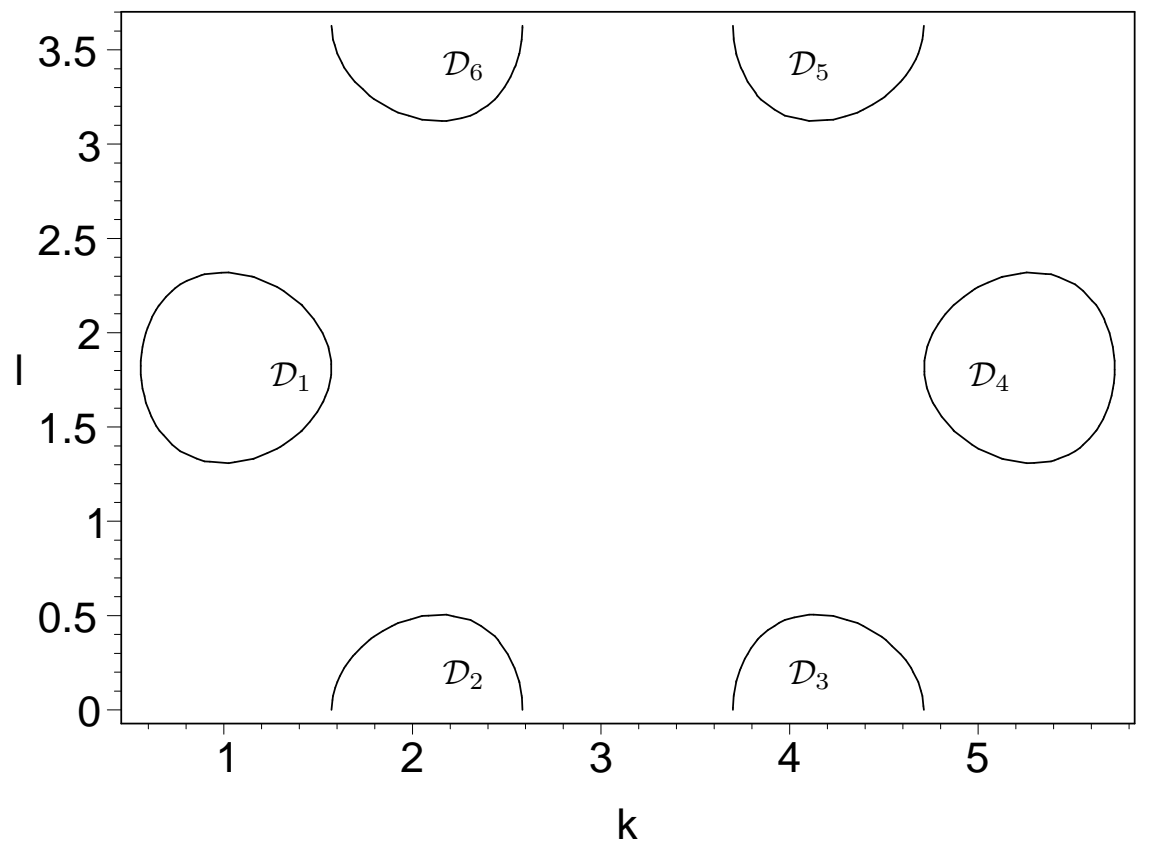

Figure 5: The domain $\mathcal{D}=\mathcal{D}_{1} \cup \ldots \cup \mathcal{D}_{6}$ in which the NLS equation (2.34) is elliptic.

maxima of $\omega(k, l)$ shown in Figure 4, namely, at the wavevectors $\left\{\mathbf{k}_{\mathbf{1}}, \ldots, \mathbf{k}_{\mathbf{6}}\right\}$ in $\mathcal{T}^{2}$; $e(k, l)$ is minimised $(e(k, l)=-48)$ at the six midpoints of the line segments which connect adjacent maxima.
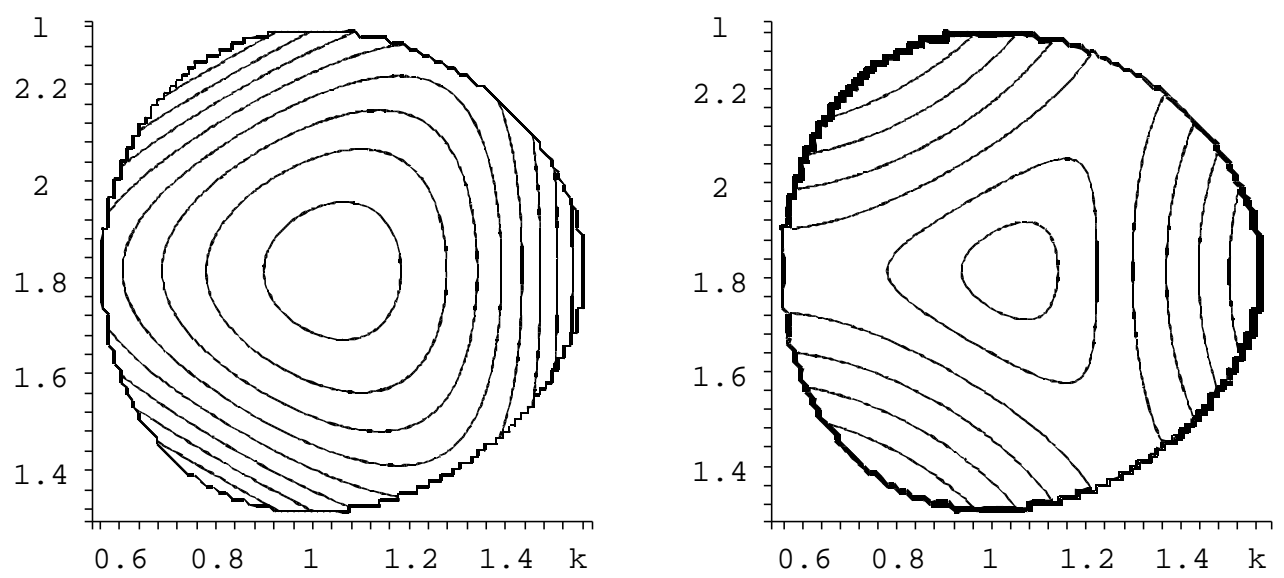

Figure 6: Contour plots of $\omega(k, l)$ (left) and speed $\left(\sqrt{u^{2}+v^{2}}\right.$, right) for wavevectors $\mathbf{k} \in \mathcal{D}_{1}$. Contours are for $2.82 \leq \omega \leq 3$ in steps of 0.02 and speeds from zero to 0.6 in steps of 0.1 . The central point corresponds to $\omega=3$ and zero speed, as one considers wave vectors nearer to the edge of $\mathcal{D}_{1}$, the frequency $\omega$ decreases and the speed increases.

Figure 6 shows a contour plot for the frequency $\omega$ in $\mathcal{D}_{1}$ : the ellipticity constraint only permits breathers with a relatively high frequency, that is, with $\omega>2.82$. This constraint in turn implies that not all breather envelope velocities are attainable, only 
breathers with speeds upto about 0.7 lattice sites per second are permitted; the plot on the right of Figure 6 shows that only breathers with speeds upto about 0.3 sites per second can move in any direction. There is then an intermediate range of speeds, between 0.3 and 0.7 sites per second where breathers can only move in certain directions, these correspond to the lattice directions. The larger the speed, the more restricted is the direction of motion.

2.6.2. Breather energy. To calculate the leading-order energy $E_{0}$ of moving breathers in lattices with a symmetric potential we use (2.37) which we write as

$$
Q_{m, n}(t) \sim 2 \varepsilon \alpha \cos \Phi \operatorname{sech}(\beta r),
$$

where $\Phi=k m+l h n+\Omega t$ is the phase of the carrier wave, $(k, l) \in \mathcal{D}, \Omega=\omega+\varepsilon^{2} \lambda$ is the breather frequency including the first correction term, $(\psi=k m+l h n+\omega t$ is only the leading-order expression). $\lambda$ parameterises the breather amplitude, $\alpha=\alpha(\lambda), \beta=\beta(\lambda)$ and $r^{2}$ are as described in equations (2.37) and (2.38).

We now find expressions for the currents $I_{m, n}, J_{m, n}$ and $K_{m, n}$ : the current $I_{m, n}$ is obtained by substituting the expression for $Q_{m, n}(2.39)$ into (2.30) and integrating with respect to time. Owing to the complexity of the expression for $r$ given by (2.38), the left-hand side of (2.30) can not be integrated with respect to time. However, the variable $r$ varies more slowly in time than $\Phi$. Hence, integration by parts (using $\left.\int f^{\prime}(t) g(\varepsilon t) \mathrm{d} t=[f(t) g(\varepsilon t)]-\varepsilon \int f(t) g^{\prime}(\varepsilon t) \mathrm{d} t\right)$ gives, to leading-order,

$I_{m, n} \sim \frac{2 \varepsilon \alpha}{\omega}[1-\cos (2 k)] \sin \Phi \operatorname{sech}(\beta r)-\frac{2 \varepsilon \alpha}{\omega} \sin (2 k) \cos \Phi \operatorname{sech}(\beta r)$,

where we have taken the constant of integration to be zero, and $\Omega \sim \omega$ to leading order. Similarly, substituting for $Q_{m, n}$ in equations (2.31) and (2.32) and integrating, we find $J_{m, n} \sim \frac{2 \varepsilon \alpha}{\omega}[1-\cos (k-l h)] \sin \Phi \operatorname{sech}(\beta r)-\frac{2 \varepsilon \alpha}{\omega} \sin (k-l h) \cos \Phi \operatorname{sech}(\beta r)$,

$K_{m, n} \sim \frac{2 \varepsilon \alpha}{\omega}[1-\cos (k+l h)] \sin \Phi \operatorname{sech}(\beta r)-\frac{2 \varepsilon \alpha}{\omega} \sin (k+l h) \cos \Phi \operatorname{sech}(\beta r)$.

Substituting these expressions into (2.29), we obtain

$$
\begin{aligned}
E_{0} \sim \sum_{m, n} & \frac{2 \varepsilon^{2} \alpha^{2}}{C_{0}} \cos ^{2} \Phi \operatorname{sech}^{2}(\beta r) \\
& +\frac{2 L \varepsilon^{2} \alpha^{2}}{\omega^{2}} \operatorname{sech}^{2}(\beta r)\left\{[(1-\cos (2 k)) \sin \Phi-\sin (2 k) \cos \Phi]^{2}\right. \\
& +[(1-\cos (k-l h)) \sin \Phi-\sin (k-l h) \cos \Phi]^{2} \\
& \left.+[(1-\cos (k+l h)) \sin \Phi-\sin (k+l h) \cos \Phi]^{2}\right\}
\end{aligned}
$$

We replace the sum by an integral since the variables $Z=\varepsilon(m-u t)$ and $W=\varepsilon(h n-v t)$ vary slowly with $m$ and $n$. To simplify the resulting integral, we approximate the terms $\cos ^{2} \Phi, \sin ^{2} \Phi$ and $\sin \Phi \cos \Phi$ by their average values of $\frac{1}{2}, \frac{1}{2}$ and 0 respectively. Hence (2.43) becomes

$$
E_{0} \sim \sum_{m, n} \frac{2 \varepsilon^{2} \alpha^{2}}{C_{0}} \operatorname{sech}^{2}(\beta r) .
$$


From the definition of $r$ (2.38) we note the function $\operatorname{sech}(\beta r)$ is not in general radially symmetric in $m, n$. Hence we work in $(\xi, \eta)$-space to facilitate evaluation of the double integral which approximates the double sum in (2.44). Evaluating the Jacobian associated with the transformation from $(m, n)$ to $(\xi, \eta)$ coordinates, we find

$$
E_{0} \sim \frac{\alpha^{2}}{h^{3} C_{0}} \sqrt{4 D_{1} D_{2}-D_{3}^{2}} \iint \operatorname{sech}\left(\beta \sqrt{\xi^{2}+\eta^{2}}\right) \mathrm{d} \xi \mathrm{d} \eta
$$

Evaluating this and substituting for $\alpha$ and $\beta$ in terms of $D=3 / 2 \omega(k, l)$ and $B=$ $3 b \omega(k, l) / 2$ (see the appendix) we find

$$
E_{0} \sim \frac{4 \pi \log 2(2 \log 2+1)}{3 h^{3} C_{0} b \omega^{2}(4 \log 2-1)} \sqrt{4 D_{1} D_{2}-D_{3}^{2}} .
$$

It is evident from (2.46) that the leading-order energy $E_{0}$ is independent of the breather amplitude, again confirming the existence of a minimum energy of moving breathers in the two-dimensional HETL with symmetric potential. However, the threshold energy does depend upon the wavenumbers $k$ and $l$ hence moving breathers have a different threshold energy. A plot of the expression (2.46) is shown in Figure 7. We see that $E_{0}$, given by (2.46), is strictly positive in the region of ellipticity $\mathcal{D}$, and is maximised (attaining the same value) at each of the points corresponding to wavevectors $\left\{\mathbf{k}_{\mathbf{1}}, \ldots, \mathbf{k}_{\mathbf{6}}\right\}$, that is, at the points which correspond to stationary breathers. The threshold energy (2.46) decays to zero towards the boundary of the elliptic domain $\mathcal{D}$ (see Figure 5). Hence, as for breathers in the square lattice, the energy threshold for moving breathers is lower than that for stationary breathers. The threshold becomes arbitrarily small at the boundary of the domain of ellipticity.

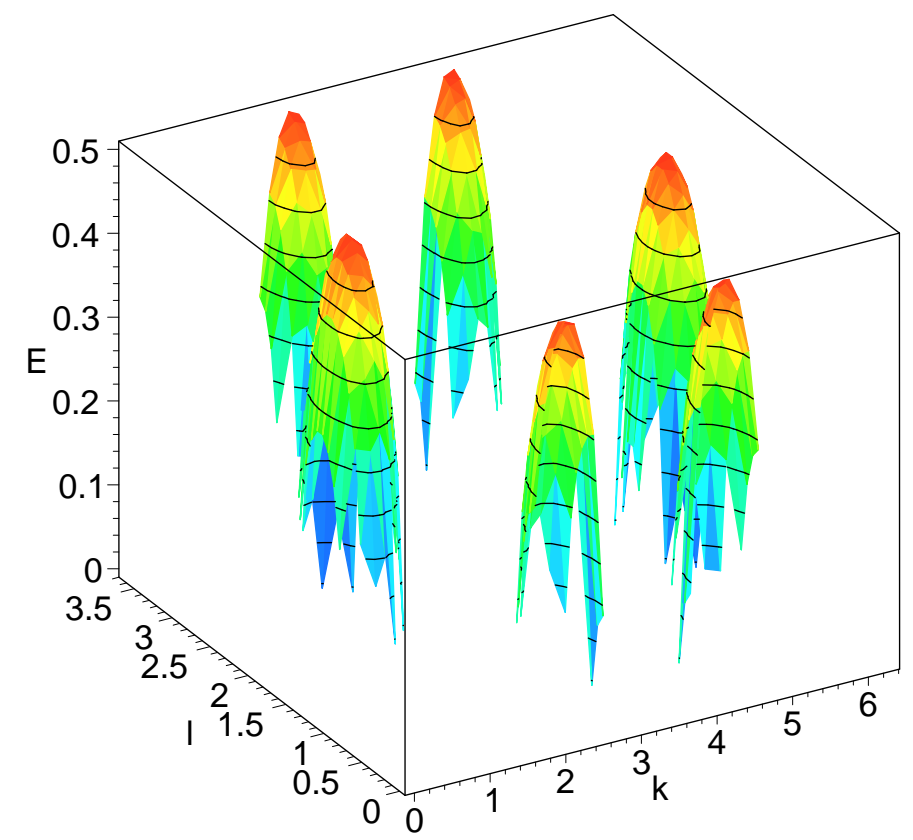

Figure 7: Plot of $E_{0}(k, l)$ for lattices with a symmetric potential. 


\subsection{Lattices with an asymmetric potential}

In this section, we consider the more general scenario where the potential $\Upsilon^{\prime}(Q)$ is asymmetric. In this case, the terms $a$ and $c$ in (2.9) and (2.10) are not both zero. If (2.21) is to be reduced to an NLS equation in $F$, both $G_{0}$ and $G_{2}$ must be found in terms of $F$. Whilst $G_{2}$ is given by the simple algebraic equation (2.20); in order to find $G_{0}$, the partial differential equation (2.23) must be solved. We assume that $G_{0}$ travels at the same velocity as $F$, that is, $G_{0}(X, Y, \tau, T) \equiv G_{0}(Z, W, T)$. Eliminating $G_{0 \tau \tau},(2.23)$ becomes

$$
\left(u^{2}-6\right) G_{0 Z Z}+\left(v^{2}-2 h^{2}\right) G_{0 W W}+2 u v G_{0 Z W}=6 a|F|_{Z Z}^{2}+2 a h^{2}|F|_{W W}^{2} .
$$

For general $k$ and $l$, it is difficult to solve for $G_{0}$ explicitly. However, for any of the wavevectors $\left\{\mathbf{k}_{\mathbf{1}}, \ldots, \mathbf{k}_{\mathbf{6}}\right\}$, the velocities $u$ and $v$ become zero, and so (2.47) becomes $\nabla^{2} G_{0}=-a \nabla^{2}|F|^{2}$, where the operator $\nabla^{2}$, defined by $\nabla_{(Z, W)}^{2} \equiv \partial_{Z}^{2}+\partial_{W}^{2}$, is equivalent to $\nabla_{(X, Y)}^{2} \equiv \partial_{X}^{2}+\partial_{Y}^{2}$. It follows that $G_{0}=-a|F|^{2}$ for each of these wavevectors.

Equations (2.18)-(2.23) are the same whichever of the wavevectors $\left\{\mathbf{k}_{\mathbf{1}}, \ldots, \mathbf{k}_{\mathbf{6}}\right\}$ is used. Thus, (2.18) gives $\omega=3$, and (2.20) implies $G_{2}=a F^{2} / 3$. Substituting these expressions for $G_{0}$ and $G_{2}$ in (2.21) gives the following NLS equation for asymmetric potentials for any of the wavevectors $\left\{\mathbf{k}_{\mathbf{1}}, \ldots, \mathbf{k}_{\mathbf{6}}\right\}$

$$
2 \mathrm{i} \omega F_{T}+3 \nabla^{2} F+\omega^{2}\left(3 b-\frac{10}{3} a^{2}\right)|F|^{2} F=0,
$$

The anomalous dispersion regime thus corresponds to $b>10 a^{2} / 9$. Soliton solutions are derived in the appendix; (2.48) corresponds to $D=1 / 2$ and $B=\left(9 b-10 a^{2}\right) / 2$, and also $r^{2}=X^{2}+Y^{2}$. Substituting the solution for $F$ into the lattice ansatz (2.17), along with the known expressions for $G_{0}$ and $G_{2}$ gives the following second-order formula for stationary breathers in lattices with an asymmetric potential

$$
\begin{aligned}
Q_{m, n}(t)=2 \varepsilon & \alpha \cos \left[k m+l h n+\left(\omega+\varepsilon^{2} \lambda\right) t\right] \operatorname{sech}(\beta r) \\
& +\frac{2}{3} a \varepsilon^{2} \alpha^{2} \operatorname{sech}^{2}(\beta r)\left\{\cos \left[2 k m+2 l h n+\left(2 \omega+2 \varepsilon^{2} \lambda\right) t\right]-3\right\}+\mathcal{O}\left(\varepsilon^{3}\right),
\end{aligned}
$$

where $\alpha$ and $\beta$ are determined in the appendix, $r=\sqrt{X^{2}+Y^{2}}$, and $X=\varepsilon m$ and $Y=\varepsilon h n$ in terms of the original discrete variables $m$ and $n$.

2.7.1. Breather energy. We calculate the leading-order energy $E_{0}$ of stationary breathers in lattices with an asymmetric potential. In this case, stationary breathers are given by (2.49), from which we take only the leading order term

$$
Q_{m, n}(t) \sim 2 \varepsilon \alpha \cos \Theta \operatorname{sech}(\beta r),
$$

where $\Theta=k m+l h n+\Omega t$ is the phase of the carrier wave with $(k, l)$ corresponding to one of $\left\{\mathbf{k}_{\mathbf{1}}, \ldots, \mathbf{k}_{\mathbf{6}}\right\}, \Omega=\omega+\varepsilon^{2} \lambda$ is the breather frequency, and $w=3$.

The currents $I_{m, n}, J_{m, n}$ and $K_{m, n}$ are obtained by substituting (2.50) into equations (2.30)-(2.32) and integrating with respect to time, taking the constant of integration to be zero. Thus, to leading order, we find

$$
I_{m, n} \sim \frac{3 \varepsilon \alpha}{\omega} \sin \Theta \operatorname{sech}(\beta r)-\frac{\sqrt{3} \varepsilon \alpha}{\omega} \cos \Theta \operatorname{sech}(\beta r),
$$




$$
\begin{aligned}
& J_{m, n} \sim \frac{3 \varepsilon \alpha}{\omega} \sin \Theta \operatorname{sech}(\beta r)+\frac{\sqrt{3} \varepsilon \alpha}{\omega} \cos \Theta \operatorname{sech}(\beta r), \\
& K_{m, n} \sim \frac{3 \varepsilon \alpha}{\omega} \sin \Theta \operatorname{sech}(\beta r)+\frac{\sqrt{3} \varepsilon \alpha}{\omega} \cos \Theta \operatorname{sech}(\beta r) .
\end{aligned}
$$

Substituting these expressions into (2.29) gives the leading-order energy

$$
\begin{aligned}
E_{0} \sim \sum_{m, n} \frac{2 \varepsilon^{2} \alpha^{2}}{C_{0}} & \cos ^{2} \Theta \operatorname{sech}^{2}(\beta r) \\
& +\frac{L h^{2}}{2 \omega^{2}} \varepsilon^{2} \alpha^{2}\left[3 h^{2} \sin ^{2} \Theta+2 h \sin \Theta \cos \Theta+3 \cos ^{2} \Theta\right] \operatorname{sech}^{2}(\beta r) .
\end{aligned}
$$

We approximate the term in square brackets by taking the average values of $\cos ^{2} \Theta$, $\sin ^{2} \Theta, \sin \Theta \cos \Theta$ as $\frac{1}{2}, \frac{1}{2}$ and 0 respectively.

We replace the double sum by an integral giving

$$
E_{0} \sim \frac{2 \alpha^{2}}{h C_{0}} \iint \operatorname{sech}^{2}\left(\beta \sqrt{X^{2}+Y^{2}}\right) \mathrm{d} X \mathrm{~d} Y .
$$

This can be evaluated to

$$
E_{0} \sim \frac{4 \pi \log 2}{h C_{0}} \frac{\alpha^{2}}{\beta^{2}}=\frac{8 \pi \log 2(2 \log 2+1)}{C_{0} h\left(9 b-10 a^{2}\right)(4 \log 2-1)} .
$$

Again, this estimate for energy is independent of the breather amplitude $\lambda$, demonstrating the energy threshold properties of the two-dimensional HETL; namely, the activation energy required to create a breather in the HETL is an $\mathcal{O}(1)$ quantity, irrespective of its amplitude $(2 \varepsilon \alpha \ll 1)$.

\section{Higher-order asymptotic analysis}

The cubic NLS equation exhibits blow-up, which cannot occur in a discrete system since energy is conserved, and even if all the energy were localised at a single site, the amplitude would still be finite. The two-dimensional cubic NLS equation description of the breather envelope is lacking. Higher-order dispersive and nonlinear effects play an important role in the dynamics of such discrete systems and therefore must be incorporated. In this section we extend our analysis of the lattice equations (2.10) to fifth-order, and derive a generalised NLS equation which includes higher-order dispersive and nonlinear terms. It then remains to determine whether this generalised NLS equation supports stable soliton solutions.

Due to the complexity of a fifth-order analysis of a general asymmetric potential, we consider only lattices with a symmetric potential, that is, those for which $a=c=0$ in (2.10). Since no second or fourth harmonic terms are generated by the nonlinearity, we use a much simpler ansatz, namely

$$
Q_{m, n}(t)=\varepsilon \mathrm{e}^{\mathrm{i} \psi} F(X, Y, \tau, T)+\varepsilon^{3} \mathrm{e}^{3 \mathrm{i} \psi} H_{3}(X, Y, \tau, T)+\cdots+\text { c.c. }
$$

where the phase $\psi=k m+l h n+\omega t$. In this case, in addition to the equations (2.24)(2.26), (2.34) and (2.22), we also have 


$$
\begin{aligned}
& \mathcal{O}\left(\varepsilon^{5} \mathrm{e}^{\mathrm{i} \psi}\right): \\
& F_{T T}= \frac{1}{6}[8 \cos (2 k)+\cos k \cos (l h)] F_{X X X X}+h^{2} \cos k \cos (l h) F_{X X Y Y} \\
&+\frac{1}{6} h^{4} \cos k \cos (l h) F_{Y Y Y Y}-\frac{2}{3} h \sin k \sin (l h) F_{X X X Y}-\frac{2}{3} h^{3} \sin k \sin (l h) F_{X Y Y Y} \\
&+6 b[2 \cos (2 k)+\cos k \cos (l h)]\left(|F|^{2} F\right)_{X X}+6 b h^{2} \cos k \cos (l h)\left(|F|^{2} F\right)_{Y Y} \\
&-12 b \sin k \sin (l h)\left[2 h F F_{X} \bar{F}_{Y}+2 h F F_{Y} \bar{F}_{X}+2 h \bar{F} F_{X} F_{Y}+h F^{2} \bar{F}_{X Y}+2 h F \bar{F} F_{X Y}\right] \\
&-3 b \omega^{2} \bar{F}^{2} H_{3}-10 \omega^{2} d|F|^{4} F .
\end{aligned}
$$

We simplify this by restricting attention to stationary breathers. Accordingly, only one extra timescale, $T=\varepsilon^{2} t$, is required, and we fix the wavenumbers $k$ and $l$ to correspond to one of the wavevectors $\left\{\mathbf{k}_{\mathbf{1}}, \ldots, \mathbf{k}_{\mathbf{6}}\right\}$. Hence we obtain the equations:

$\mathcal{O}\left(\varepsilon^{3} \mathrm{e}^{\mathrm{i} \psi}\right)$

$$
2 \mathrm{i} \omega F_{T}+3 \nabla^{2} F+3 b \omega^{2}|F|^{2} F=0
$$

$\mathcal{O}\left(\varepsilon^{5} \mathrm{e}^{\mathrm{i} \psi}\right)$

$$
F_{T T}=-\frac{3}{4} \nabla^{4} F-9 b \nabla^{2}\left(|F|^{2} F\right)-10 \omega^{2} d|F|^{4} F,
$$

in addition to $\omega=3$ (from the $\mathcal{O}\left(\varepsilon \mathrm{e}^{\mathrm{i} \psi}\right)$ equation) and $H_{3}=0$ (from $\mathcal{O}\left(\varepsilon^{3} \mathrm{e}^{3 \mathrm{i} \psi}\right)$ ). A consequence of the hexagonal symmetry of the HETL is that all differentials on the right-hand side of (3.4) are isotropic.

In order to obtain a generalised NLS equation, we combine the higher-order equation (3.4) with the cubic two-dimensional NLS equation (3.3). The $F_{T T}$ term on the left-hand side of (3.4) is eliminated by differentiating (3.3) with respect to $T$ and substituting for $F_{T T}$ into (3.4). The resulting expression is

$$
\begin{aligned}
6 \mathrm{i} F_{T}+3 \nabla^{2} F+27 b|F|^{2} F+\frac{\varepsilon^{2}}{2} \nabla^{4} F+\frac{9 \varepsilon^{2}}{4}\left(40 d-27 b^{2}\right)|F|^{4} F \\
+\frac{27 b \varepsilon^{2}}{4} \nabla^{2}\left(|F|^{2} F\right)-\frac{9 b \varepsilon^{2}}{2}|F|^{2} \nabla^{2} F-\frac{9 b \varepsilon^{2}}{4} F^{2} \nabla^{2} \bar{F}=0
\end{aligned}
$$

which is isotropic.

To the best of our knowledge, this perturbed form of the NLS equation has not been studied in the literature before; although it is similar to and slightly simpler than the corresponding equation derived for the square lattice [2]. It is also similar to the perturbed NLS equation considered by Davydova et al [4], namely

$$
\mathrm{i} F_{T}+D \nabla^{2} F+B|F|^{2} F+P \nabla^{4} F+K|F|^{4} F=0,
$$

which is known to have stable soliton solutions. The anomalous dispersion case is $B D>0$, which in our equation (3.5) corresponds to $b>0$; Davydova's criteria for soliton existence is $P K>0$ which implies $40 d>27 b^{2}$. Hence, it is in this parameter regime that we seek breathers solutions of the HETL. The numerical results presented in 
Section 4 show that long-lived breather solutions are supported by the two-dimensional hexagonal FPU lattice, suggesting that the additional perturbing terms in (3.5) do not destabilise the Townes soliton. In fact, numerical simulations (presented in [1] but not here) of the case $40 d<27 b^{2}$ show the breather mode to be long-lived, suggesting that the terms on the second line of (3.5) are stabilising.

We have been unable to find a variational formulation of (3.5), and are therefore unable to use the methods of Davydova et al. [4] and Kuznetsov et al. [13]. Alternative possible methods include the modulation theory of Fibich and Papanicolaou [6, 7].

\section{Numerical results}

\subsection{Preliminaries}

Using a fourth-order Runge-Kutta scheme, we solve the equations

$$
\begin{aligned}
& \frac{\mathrm{d} Q_{m, n}}{\mathrm{~d} t}=R_{m, n} \\
& \frac{\mathrm{d} R_{m, n}}{\mathrm{~d} t}=\left(\delta_{I}^{2}+\delta_{J}^{2}+\delta_{K}^{2}\right)\left[Q_{m, n}+a Q_{m, n}^{2}+b Q_{m, n}^{3}+c Q_{m, n}^{4}+d Q_{m, n}^{5}\right],
\end{aligned}
$$

numerically. Introducing the variable $R_{m, n}$ converts the system of second-order ordinary differential equations (2.10) to an equivalent system of first-order differential equations.

We present the results of simulations for a range of parameter values. From (2.26), the velocity of the envelope $(u, v)$ depends upon the wavevector $\mathbf{k}=[k, l]^{T}$, and hence we obtain moving breathers by choosing $(k, l) \in \mathcal{D}$. In Figure 8 , we show the points in $\mathcal{D}_{1} \subset \mathcal{D}$ for which we solve the lattice equations (4.1) numerically. These points correspond to the wavevectors $\mathbf{k}_{\mathbf{1}}=[\pi / 3, \pi / h]^{T}, \mathbf{k}_{\mathbf{a}}=[1.4, \pi / h]^{T}, \mathbf{k}_{\mathbf{b}}=[0.79,1.7324]^{T}$ and $\mathbf{k}_{\mathbf{c}}=[0.8,1.9987]^{T}$. Selecting $(k, l)$ too near to the boundary of $\mathcal{D}$ results in a sharply elongated breather, which are difficult to simulate as they require a large domain. Wavevectors close to any of $\left\{\mathbf{k}_{\mathbf{1}}, \ldots, \mathbf{k}_{\mathbf{6}}\right\}$, lead to breather modes with small speeds, a check of breather velocity would then require a long-time simulation. In practice we have chosen wavenumbers which do not lead to a severely elongated breather envelope, and yet have velocities which result in observable displacements over reasonable times. We present simulations of stationary breathers in systems with asymmetric potentials $(a, c \neq 0)$ in Section 4.8 and, in Sections 4.4-4.7, simulations of stationary and moving breathers in lattices with symmetric potentials (that is, $a=c=0$ in (4.1)).

\subsection{Initial data and boundary conditions}

We generate initial data by using the analytic expressions for breather solutions derived in Section 2.3. The formulae for $Q_{m, n}$ and $R_{m, n}$ are found in terms of the original discrete variables $m$ and $n$, and then shifted horizontally and vertically so that initially the breather lies at the centre of the lattice. We impose periodic boundary conditions for the lattice in both horizontal and vertical directions, converting the two-dimensional arrangement illustrated in Figure 1 into a two-torus. In long-time 


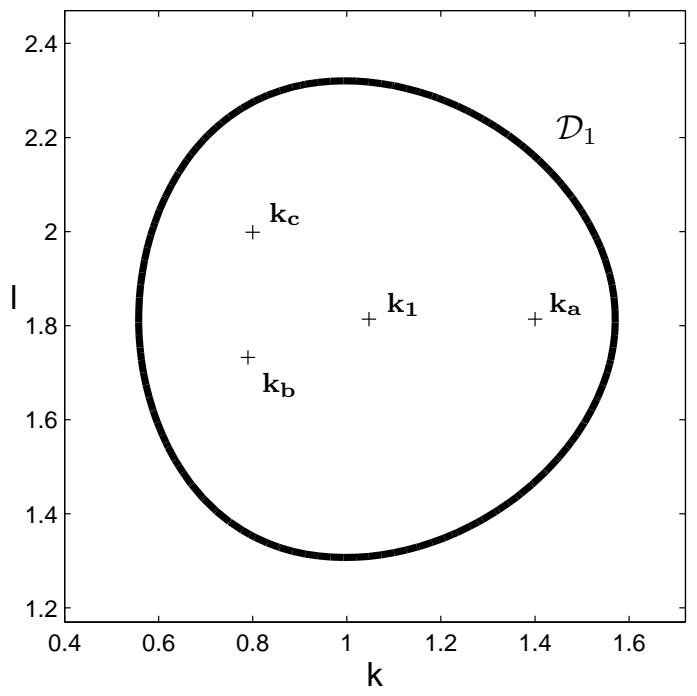

Figure 8: Wavevectors in $\mathcal{D}_{1} \subset \mathcal{D}$ for which breathers are simulated.

simulations, moving breathers which approach an edge of the lattice reappear from the opposite edge. We select the site $(1,1)$ to lie at the bottom left-hand corner of the arrangement as illustrated in Figure 9. Sites along the boundaries and corners are missing between one and four neighbours. We introduce fictitious sites along the boundaries and corners where necessary to effect periodic boundary conditions.

An illustration of a small finite lattice is shown in Figure 9. The dots represent capacitors located at lattice sites, and the lines represent some of the inter-connecting inductors. From equations (4.1), the charge $Q_{m, n}$ stored on each capacitor depends upon the charge stored on the capacitors located at its six neighbouring sites, two in each of the directions $\mathbf{e}_{\mathbf{i}}, \mathbf{e}_{\mathbf{j}}$ and $\mathbf{e}_{\mathbf{k}}$. For the sake of clarity, the inductors connecting the capacitors at the center of each hexagon to its six nearest neighbours are not shown (see Figure 1). It is not necessary that the lattice should be "square," meaning that the lattice could comprise $M \times N$ lattice sites, with $M \neq N$. However, typically, we consider $M=N$ as in Figure 9, since the numerical routines are simpler to encode when the lattice $i s$ square, and in the examples below, we consider lattices with $N \leq 50$.

\subsection{Numerical computation of breather energy}

Since the HETL is lossless the total energy is conserved and can be used as a check of the accuracy of our numerical scheme. We compute the leading-order energy by expressing the summand $e_{m, n}^{(0)}(2.29)$ in terms of the output variables of the numerical routine, namely $Q_{m, n}$ and $R_{m, n}$. The first term of $e_{m, n}^{(0)}$ is simply dependent on $Q_{m, n}$. It remains to find the currents $I_{m, n}, J_{m, n}$ and $K_{m, n}$ in terms of $Q_{m, n}$ and $R_{m, n}$. The details of this calculation depend upon whether the interaction potential is symmetric or asymmetric. As a check of the numerical scheme we verify that the sum is conserved 


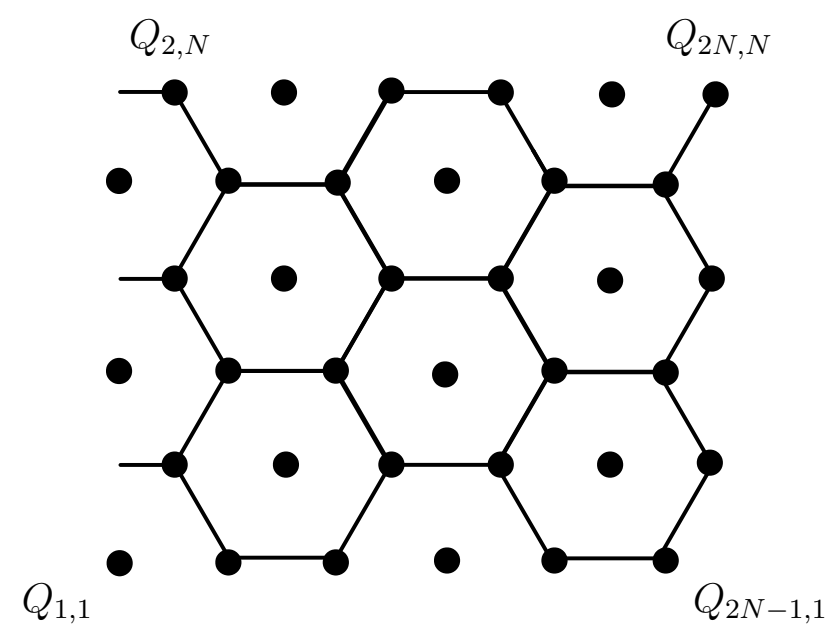

Figure 9: Periodic boundary conditions for the two-dimensional HETL.

and that it agrees with the asymptotic estimate (2.46).

4.3.1. Lattices with a symmetric potential. First, we find the current $I_{m, n}$ in terms of $Q_{m, n}$ and $R_{m, n}$. Differentiating the leading-order expression for the breather given by (2.39), we have (retaining leading-order terms only)

$$
\dot{Q}_{m, n}=R_{m, n} \sim-2 \varepsilon \alpha \omega \sin \Phi \operatorname{sech}(\beta r),
$$

where $\alpha, \beta$ and $r^{2}$ are as defined in Section 2.6. Comparing the analytic expression (2.40) for the current $I_{m, n}$ with the expressions for $Q_{m, n}(2.39)$ and $R_{m, n}$ (4.2), we find

$$
I_{m, n}=\frac{\cos (2 k)-1}{\omega^{2}} R_{m, n}-\frac{\sin (2 k)}{\omega} Q_{m, n} .
$$

Similarly, comparing the expressions $(2.41)$ and (2.42) for the currents $J_{m, n}$ and $K_{m, n}$ respectively with equations (2.39) and (4.2), we find

$$
\begin{aligned}
& J_{m, n}=\frac{\cos (k-l h)-1}{\omega^{2}} R_{m, n}-\frac{\sin (k-l h)}{\omega} Q_{m, n}, \\
& K_{m, n}=\frac{\cos (k+l h)-1}{\omega^{2}} R_{m, n}-\frac{\sin (k+l h)}{\omega} Q_{m, n} .
\end{aligned}
$$

Substituting these expressions into (2.29), gives an expression for $E_{0}$ which does not simplify, so we do not reproduce it here.

4.3.2. Lattices with an asymmetric potential. Differentiating the leading-order expression (2.50) for the charge $Q_{m, n}$ in lattices with an asymmetric potential gives

$$
\dot{Q}_{m, n}=R_{m, n} \sim-2 \varepsilon \alpha \omega \sin \Theta \operatorname{sech}(\beta r),
$$


where $\Theta=\pi m / 3+\pi n+\Omega t$ with $\Omega=3$. Using (2.50) and (4.6), the current $I_{m, n}$ given by (2.51) can be expressed in terms of $Q_{m, n}$ and $R_{m, n}$ as

$$
I_{m, n} \sim-\frac{\sqrt{3}}{2 \omega}\left[\frac{\sqrt{3} R_{m, n}}{\omega}+Q_{m, n}\right] .
$$

Similarly, from (2.52)-(2.53), we have

$$
J_{m, n}=K_{m, n} \sim-\frac{\sqrt{3}}{2 \omega}\left[\frac{\sqrt{3} R_{m, n}}{\omega}-Q_{m, n}\right] .
$$

These are equivalent to substituting $k=\pi / 3$ and $l=\pi / h$ into (4.3)-(4.5). Inserting the expressions for $I_{m, n}, J_{m, n}$ and $K_{m, n}((4.7)-(4.8))$ into (2.29) yields

$$
E_{0}=\sum_{m, n} \frac{1}{72 C_{0}}\left[45 Q_{m, n}^{2}+3 R_{m, n}^{2}-2 \sqrt{3} Q_{m, n} R_{m, n}\right] .
$$

4.3.3. Effective breather width. Numerical computation of the energy as described above allows us to check that the total lattice energy is conserved, but does not indicate whether a breather changes shape over time. To remedy this, we define breather widths in the $m$ and $n$ directions, the sum of which we denote $\mathcal{W}_{\mathrm{br}}$, where

$$
\mathcal{W}_{\mathrm{br}}^{2}=\frac{r_{20}}{E_{0}}+\frac{r_{02}}{E_{0}}-\left(\frac{r_{10}}{E_{0}}\right)^{2}-\left(\frac{r_{01}}{E_{0}}\right)^{2}
$$

and $r_{10}, r_{01}, r_{20}$ and $r_{02}$ are defined by

$$
\begin{aligned}
r_{10} & =\sum_{m, n} m e_{m, n}, & r_{20} & =\sum_{m, n} m^{2} e_{m, n}, \\
r_{01} & =\sum_{m, n} h n e_{m, n}, & r_{02} & =\sum_{m, n} h^{2} n^{2} e_{m, n} .
\end{aligned}
$$

The variation in $\mathcal{W}_{\mathrm{br}}$ over time gives a measure of the distortion suffered by a breather.

\subsection{Stationary breather in a lattice with symmetric potential}

We investigate stationary breather solutions in the anomalous dispersion regime which corresponds to $b>0$ (see (3.5)). We set $k=\pi / 3$ and $l=\pi / h$, corresponding to $\mathbf{k}_{\mathbf{1}}$ in Figure 8, so that the velocities $u$ and $v$ are zero. Davydova's result implies that we expect to find stable soliton solutions when $P K>0$. From (3.6), $P=\varepsilon^{2} / 2$, hence this inequality implies $40 d>27 b^{2}$. Hence we choose $b=d=1$ as well as $N=30, \varepsilon=0.2$ and $\lambda=1$. Using the technique outlined in the appendix we calculate $\alpha=1.0212$, $\beta=1.8670$. The breather frequency is $\omega+\varepsilon^{2} \lambda=3.040$, and therefore the period of oscillation is $T=2.0668$.

As is the case for all our simulations, the initial profile of the breather is located at the centre of the lattice, as illustrated in Figure 10 (a). At $t=0$, the breather energy is $E_{0}=0.7606$, and $\mathcal{W}_{\mathrm{br}}=3.74$. The asymptotic estimate for $E_{0}$ given by (2.46) is 0.7523 , which is only $1 \%$ different from the numerically obtained value. In Figure 10 (c) we show the breather after 30 oscillations. Plots of the local energy $e_{m, n}$ at $t=0$ and $t=30 T$ are presented in Figures 10 (b) and 10 (d). After thirty oscillations the breather has shed a small amount of energy, which is manifested as small amplitude radiation throughout 
the lattice. Accordingly, the breather appears a little distorted in shape compared to its initial profile. In particular, at $t=30 T$, we find $\mathcal{W}_{\mathrm{br}}=4.21$. The energy of the breather at $t=30 T$ is 0.7087 , giving $\Delta E_{0} / E_{0}=-0.0682$,

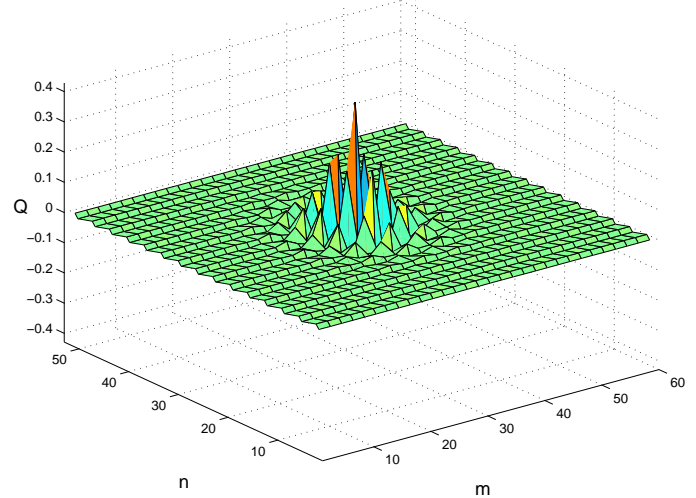

(a) Profile at $t=0$.

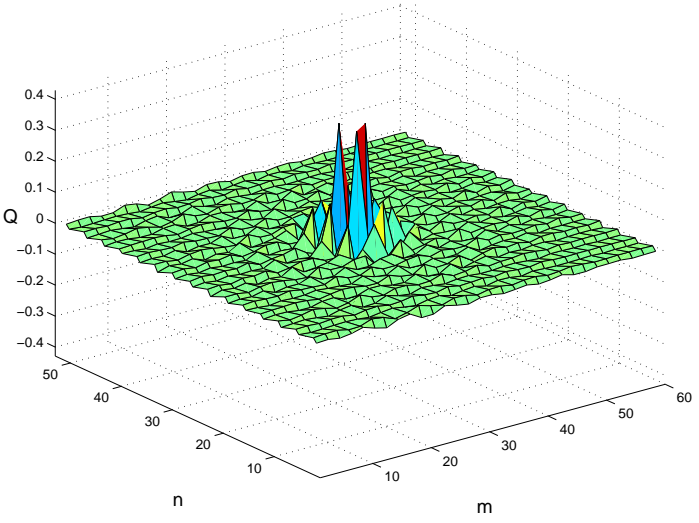

(c) Profile at $t=30 T=62.82$.

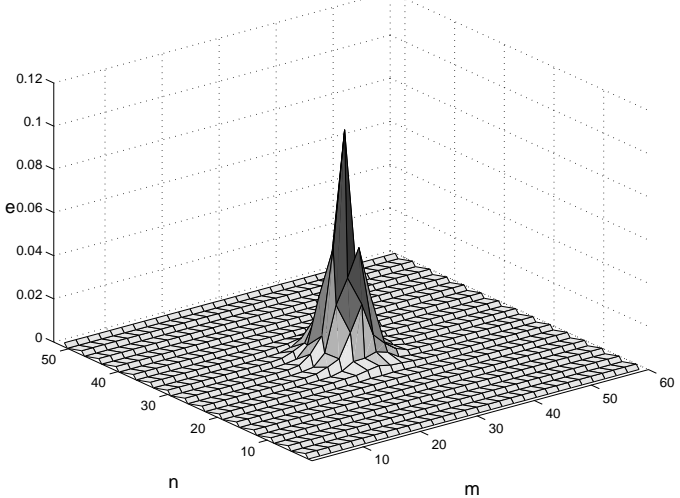

(b) Plot of $e_{m, n}, E_{0}=0.7606$.

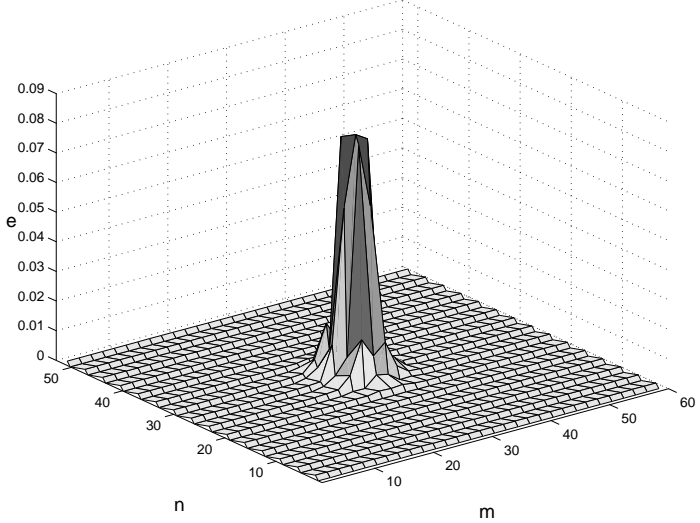

(d) Plot of $e_{m, n}, E_{0}=0.7087$.

Figure 10: Stationary breather in a lattice with symmetric potential, see Section 4.4 for details.

\subsection{Breather moving along a lattice direction $\left(\Psi=0^{\circ}\right)$}

In Figure 11, we show a simulation of a breather moving along a lattice direction parallel to the $m$-axis, that is, $\Psi=0^{\circ}$. We have chosen $\mathbf{k}=\mathbf{k}_{\mathbf{a}}=[1.4, \pi / h]^{T}$ so that $u=0.4445$ and $v=0$ and thus $\Psi=\tan ^{-1}(v / u)=0^{\circ}$. The breather frequency is $\omega=2.9265$, and hence the period $T=2.1470$. Following the calculation outlined in the appendix, the amplitude and width parameters are $\alpha=1.0339$ and $\beta=1.8440$; the remaining parameters being $b=1, d=1, N=30, \varepsilon=0.1$ and $\lambda=1$. 
The initial profile of the breather is shown in Figure $11(\mathrm{a})$, and at this time, the calculated energy is $E_{0}=0.5537$, whilst the asymptotic estimate (2.46) is $E_{0}=0.5522$. It may be observed that the breather is not radially symmetric. In fact, it is slightly elongated in the direction parallel to the $m$-axis, that is, parallel to the direction of motion. This is because the point corresponding to the wavevector $\mathbf{k}_{\mathbf{a}}$ is near the boundary of the region of ellipticity $\mathcal{D}_{1}$ as illustrated in Figure 5.

Figure 11 (b) shows the breather at the later time of $t=21.58 T=46.3513$, at which time we find $E_{0}=0.5562$. By this time, the breather has reached the right-hand edge of the lattice and, owing to periodic boundary conditions, it reemerges from the lefthand side as shown in Figure 11 (c). The breather remains localised, without spreading, though it leaves behind a small amount of energy in its path (visible in Figures 11 (b) and $11(\mathrm{c}))$. The computed energy changes much less than for the previous simulation (here, $\Delta E_{0} / E_{0}=0.0287$ ).

The velocity of the breather can be measured from a plot of the energy $e_{m, n}$, which is shown in Figure $11(\mathrm{~d})$, viewed from directly above the plane of the lattice. From this plot, we note that the breather has travelled 42 units at an average speed of 0.42 units per second, which is $5.5 \%$ lower than our predicted speed of 0.4445 units per second. This is consistent with a small amount of energy being shed as the system transforms from our approximated initial conditions into the precise shape of the breather.

\subsection{Breather moving at $\Psi=210^{\circ}$}

We now show that it is possible to simulate breathers moving in directions other than a lattice direction (that is, $\Psi \neq 0^{\circ}$ ). We set $k=0.79$ and $l=1.7324$, which corresponds to $\mathbf{k}_{\mathbf{b}}$ in Figure 8. It may be verified that $u=-0.1999$ and $v=-0.1154$ units per second, leading to $\Psi=210^{\circ}$ as required. Although not a lattice vector, this direction is an axis of symmetry of the lattice. For the wavevector $\mathbf{k}_{\mathbf{b}}$, we have $\omega=2.9675$ and hence $T=2.1174$, the remaining parameters being $b=1, d=1$, $N=30, \varepsilon=0.1$ and $\lambda=1$. The variational parameters $\alpha$ and $\beta$ are 1.0267 and 1.8568 respectively. The breather is shown at times $t=25,50,75$ and 100 seconds in Figure 12 . Clearly, the breather does not deform significantly as it travels, nor does it radiate much energy. The initial energy is computed to be $E_{0}=0.6306$, and at $t=100$, the energy is 0.6275 , a loss of $0.5 \%$. The asymptotic estimate for the energy is $E_{0}=0.6184-2 \%$ different from the numerically computed value. The motion of the breather is charted in Table 1 , the final measured values for the velocities $u$ and $v$ give an average speed of 0.2152 units per second, $6.8 \%$ below the predicted speed of 0.2308 units per second. The angle of travel is almost identical to the expected value of $\Psi=210^{\circ}$.

\subsection{Breather moving at $\Psi=130^{\circ}$}

We have presented simulations of breathers which move along axes of symmetry of the lattice. In Section 2.6.1, from the results of our asymptotic analysis, we found that breather solutions could be constructed for any direction of travel. In this section, we test 


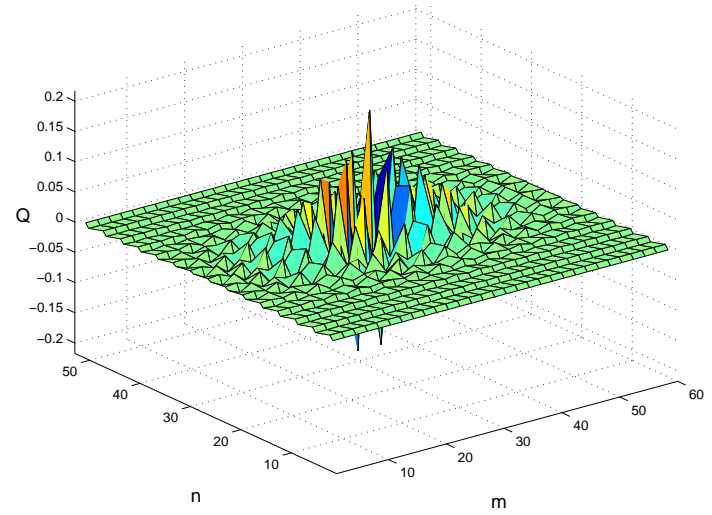

(a) Profile at $t=0, E_{0}=0.5537$.

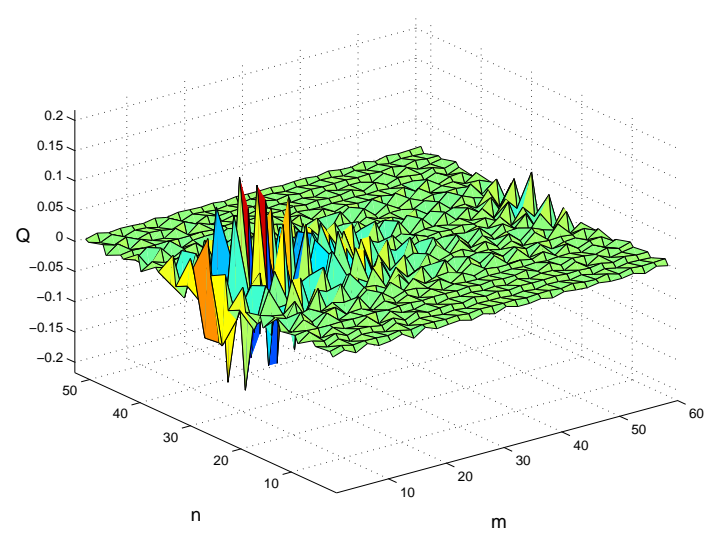

(c) Profile at $t=46.59 T, E_{0}=0.5696$.

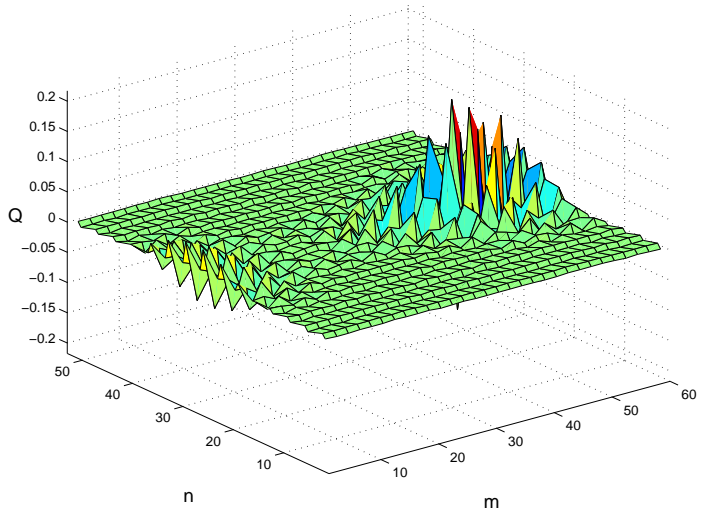

(b) Profile at 21.58T, $E_{0}=0.5562$.

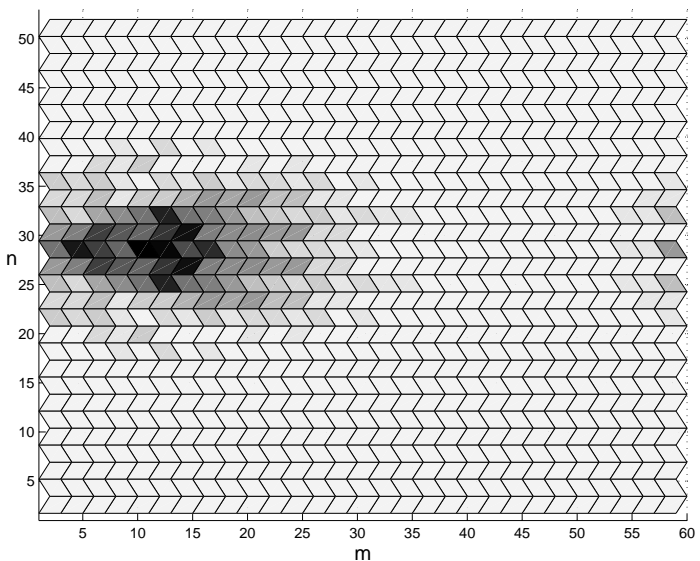

(d) Plot of $e_{m, n}$.

Figure 11: Breather moving along a lattice direction, $\Psi=0^{\circ}$, see Section 4.5 for details.

\begin{tabular}{|c|c|c|c|c|c|c|}
\hline$\underset{(s)}{\operatorname{Time}}$ & $\begin{array}{c}\text { Horizontal } \\
\text { displacement }\end{array}$ & $\begin{array}{c}\text { Vertical } \\
\text { displacement }\end{array}$ & $\begin{array}{l}\text { Average horizontal } \\
\text { velocity (units s s }\end{array}$ & $\begin{array}{c}\text { Average vertical } \\
\text { velocity }\left(\text { units s } \text { s }^{-1} \text { ) }\right.\end{array}$ & $\tan \Psi$ & $\Psi$ \\
\hline 25 & -3.5 & -2 & -0.14 & -0.08 & 0.5714 & $209.74^{\circ}$ \\
\hline 50 & -8 & -4.5 & -0.16 & -0.09 & 0.5625 & $209.36^{\circ}$ \\
\hline 75 & -13 & -7.5 & -0.1733 & -0.1 & 0.5770 & $209.99^{\circ}$ \\
\hline 100 & -18.5 & -11 & -0.185 & -0.11 & 0.5946 & $210.74^{\circ}$ \\
\hline
\end{tabular}

Table 1: Summary of breather motion $\left(\Psi=210^{\circ}\right)$.

the mobility of breathers along directions which do not correspond to axes of symmetry of the lattice. We have successfully propagated breathers in a range of directions, and here we show only one such breather, moving at an angle $\Psi=130^{\circ}$. We set $k=0.8$ and 


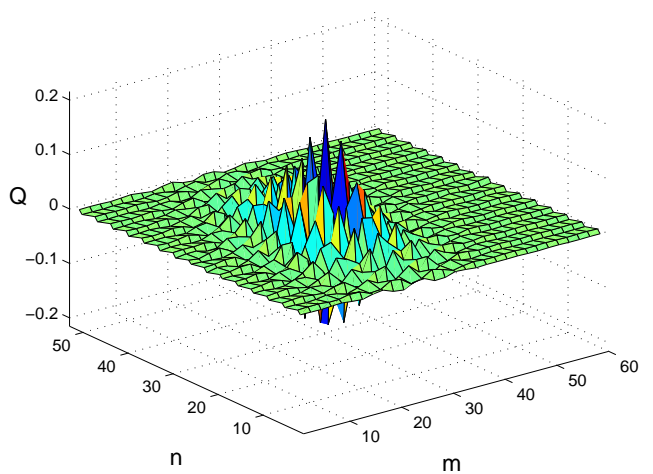

(a) Profile at $t=25, E_{0}=0.6295$.

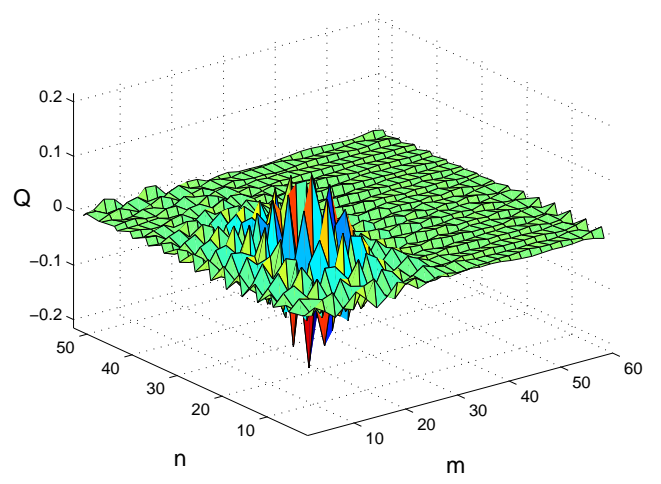

(c) Profile at $t=75, E_{0}=0.6235$.

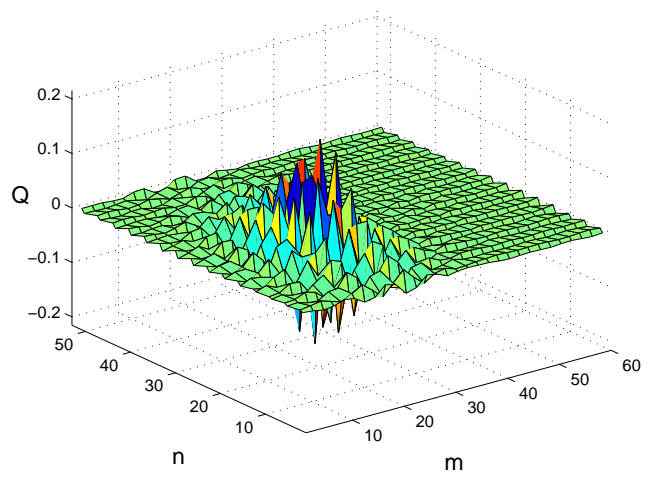

(b) Profile at $t=50, E_{0}=0.6292$.

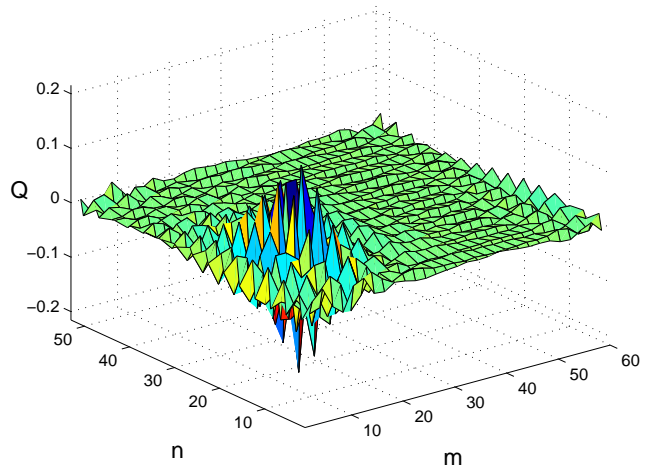

(d) Profile at $t=100, E_{0}=0.6275$.

Figure 12: Breather moving at $\Psi=210^{\circ}$, see Section 4.6 for details.

$l=1.9987$, from which we find $u=-0.2160, v=0.2575, \Psi=130^{\circ}, \omega=2.9502$ and the period is $T=2.1298$. The remaining parameters are $b=1, d=1, N=30, \varepsilon=0.1$, $\lambda=1, \alpha=1.0297$ and $\beta=1.8514$. From Figure 13, Even at 120 seconds, the breather remains localised without suffering appreciable degradation nor is much radiation left behind in its wake. Initially the energy $E_{0}$ is computed as 0.5977 , only $0.2 \%$ different from the asymptotic estimate of $E_{0}=0.5965$. After 120 seconds, $E_{0}$ is 0.5897 , a change of $1.3 \%$. To find the velocity of the breather, we have recorded its motion and presented relevant data in Table 2. From (2.26), the average speed is predicted to be 0.3254 units per second; whilst our numerical simulation shows an average speed of 0.3361 units per second, $3.2 \%$ lower than the expected speed. The direction of motion of the breather is predicted accurately. 


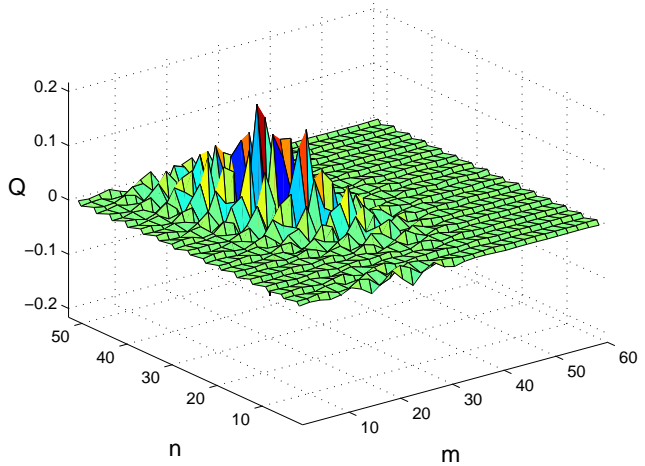

(a) Profile at $t=30, E_{0}=0.5961$.

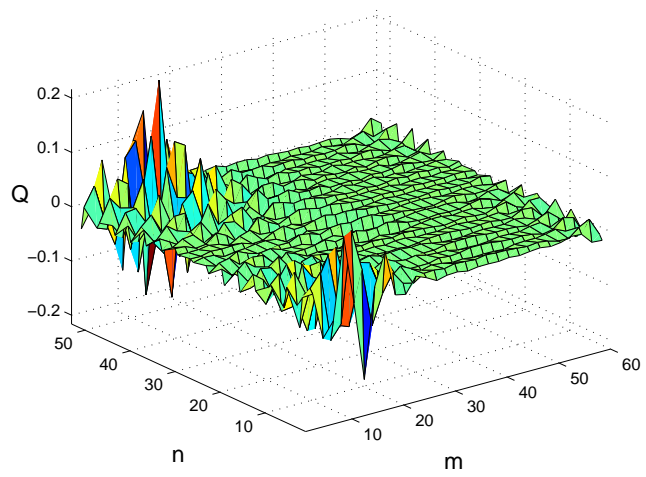

(c) Profile at $t=90, E_{0}=0.5939$.

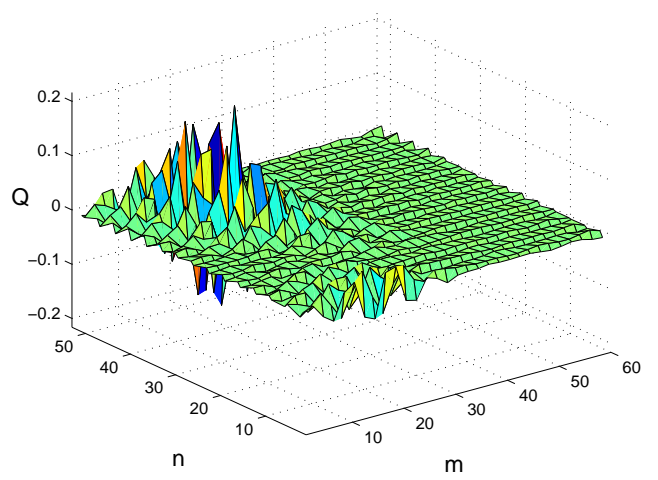

(b) Profile at $t=60, E_{0}=0.5966$.

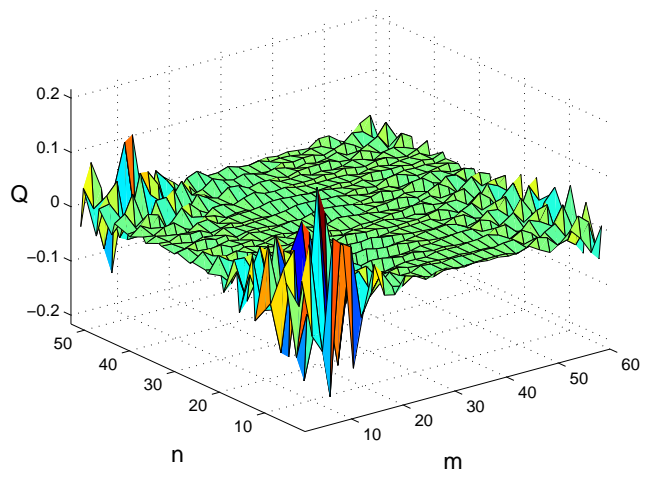

(d) Profile at $t=120, E_{0}=0.5897$.

Figure 13: Breather moving at $\Psi=130^{\circ}$, see Section 4.7 for details.

\begin{tabular}{|c|c|c|c|c|c|c|}
\hline$\underset{(s)}{\operatorname{Time}}$ & $\begin{array}{c}\text { Horizontal } \\
\text { displacement }\end{array}$ & $\begin{array}{c}\text { Vertical } \\
\text { displacement }\end{array}$ & $\begin{array}{l}\text { Average horizontal } \\
\text { velocity (units s }{ }^{-1} \text { ) }\end{array}$ & $\begin{array}{l}\text { Average vertical } \\
\text { velocity (units } \mathrm{s}^{-1} \text { ) }\end{array}$ & $\tan \Psi$ & $\Psi$ \\
\hline 30 & -6 & 7 & -0.2 & 0.2333 & -1.1667 & $130.60^{\circ}$ \\
\hline 60 & -11 & 13.5 & -0.1833 & 0.225 & -1.2273 & $129.17^{\circ}$ \\
\hline 90 & -17.5 & 21.5 & -0.1944 & 0.2389 & -1.2286 & $129.14^{\circ}$ \\
\hline 120 & -25 & 30 & -0.2083 & 0.25 & -1.2 & $129.81^{\circ}$ \\
\hline
\end{tabular}

Table 2: Summary of breather motion $\left(\Psi=130^{\circ}\right)$ see Section 4.7 for details.

\subsection{Stationary breather in a lattice with asymmetric potential}

In Sections 4.4-4.7, we have shown simulations of lattices with a symmetric potential, that is, with $a$ and $c$ not necessarily zero in (4.1). We now consider the more general case for which the interaction potential is asymmetric $(a \neq 0 \neq c)$. 
To illustrate a stationary breather, we generate initial data using (2.49), with the wavevector $\mathbf{k}=\mathbf{k}_{\mathbf{1}}=[\pi / 3, \pi / h]^{T}$. As discussed in Section 2.7, anomalous dispersion corresponds to $b>10 a^{2} / 9$, hence we choose $a=1, b=2.5, c=0$ and $d=1$. The remaining parameter values are $N=30, \varepsilon=0.1, \lambda=1$, hence $\omega=3, T=2.0668$, $\alpha=0.8665$ and $\beta=1.8670$.

The breather is shown in Figure 14 after 10, 30 and 40 full oscillations. Initially, we find the breather's width and energy are $\mathcal{W}_{\mathrm{br}}=7.46$ and $E_{0}=0.5429$, the latter being only $0.26 \%$ different from the asymptotic estimate (2.56) of 0.5416 . The accompanying plots of $e_{m, n}$ demonstrate clearly that the breather preserves its form and remains localised, even after 80 seconds. At $t=40 T$, we find that $\mathcal{W}_{\mathrm{br}}=6.83$, a narrowing of the breather by $8 \%$. Also, at $t=40 T$, the numerically computed value of the energy $E_{0}=0.5371$, showing that the energy does not fluctuate significantly.

\section{Discussion}

In this paper, we have found approximations to discrete breathers in a twodimensional hexagonal FPU lattice with a scalar-valued function at each node. We have shown that the lattice equations can be reduced to a cubic NLS equation for two special cases. In Section 2.6, we obtained moving breathers when the interaction potential is symmetric; in this case an ellipticity criterion for the wavevector is found. In the appendix we summarise our technique for approximating soliton solutions of the two-dimensional NLS equation.

In Section 2.7, we considered lattices with an asymmetric potential, in which case a reduction to NLS could only be performed for stationary breathers. The theoretical methods employed here are similar to those used on the square lattice of [2]; however, several important differences emerge in the course of the analysis. We found that the anomalous dispersion regime corresponds to $b>4 a^{2} / 3$ in the square lattice and $b>10 a^{2} / 9$ in the hexagonal. Furthermore we find that stationary breathers involve the generation of second harmonics in the hexagonal lattice $\left(G_{2}=a F^{2} / 3\right)$, whereas they are suppressed in the square lattice $\left(G_{2}=0\right)$. In both lattices, the quadratic nonlinearity generates a small amplitude slowly-varying mode $\left(G_{0}=-a|F|^{2}\right)$.

For symmetric interactions we found an associated ellipticity constraint: moving breathers only occur for certain wavevectors, this means that all breathers have (i) a relatively high frequency, and (ii) a maximum speed, which depends on the wave vector and hence on the direction of travel, (iii) a threshold energy which also depends on wavevector and hence is related to speed and direction of travel. We find that the threshold energy is lower for breathers at the edge of the domain of ellipticity in wavevector space. These breathers have lower frequencies and faster speeds. The fastest-moving breathers are restricted to moving along lattice directions.

We also presented asymptotic estimates for the breather energy in Sections 2.6 and 2.7. As expected, we found a minimum energy required to create breathers in the hexagonal lattice. The threshold energy for moving breathers is smaller than that 


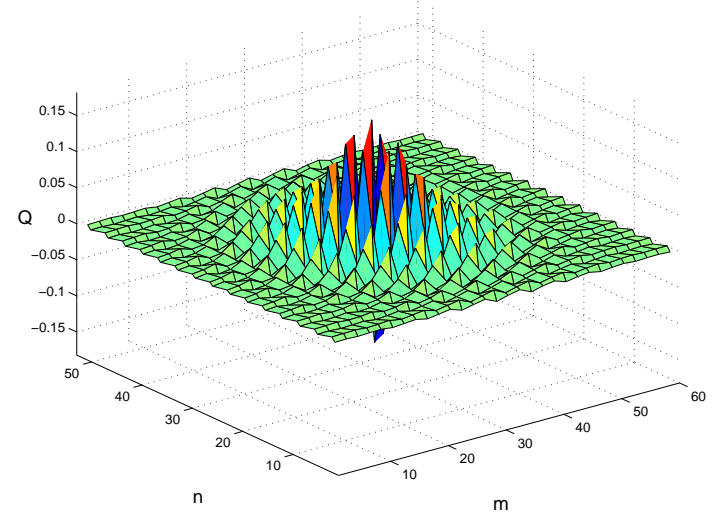

(a) $t=10 T=20.69$.

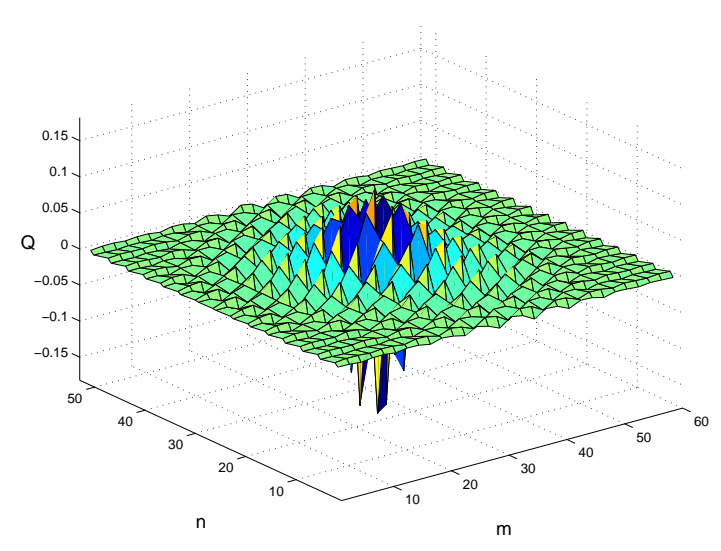

(c) $t=30 T=41.34$.

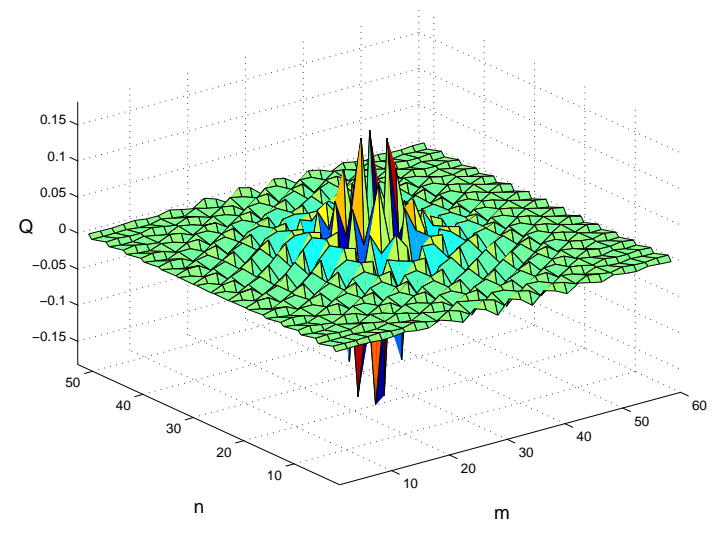

(e) $t=40 T=82.67$.

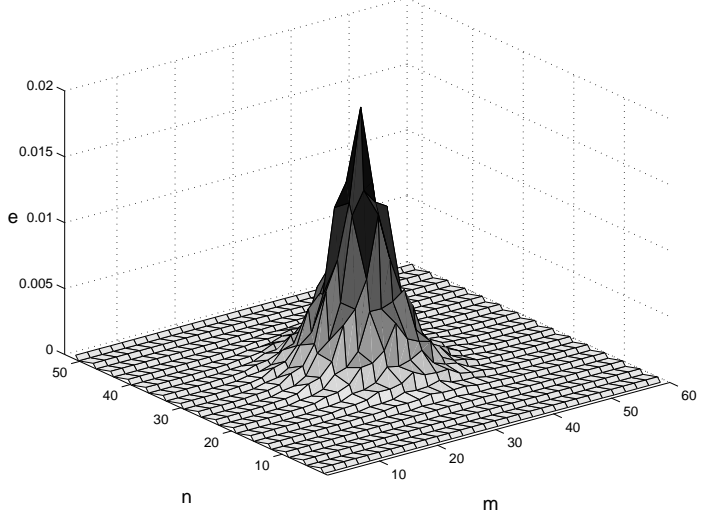

(b) Plot of $e_{m, n}, E_{0}=0.5453$.

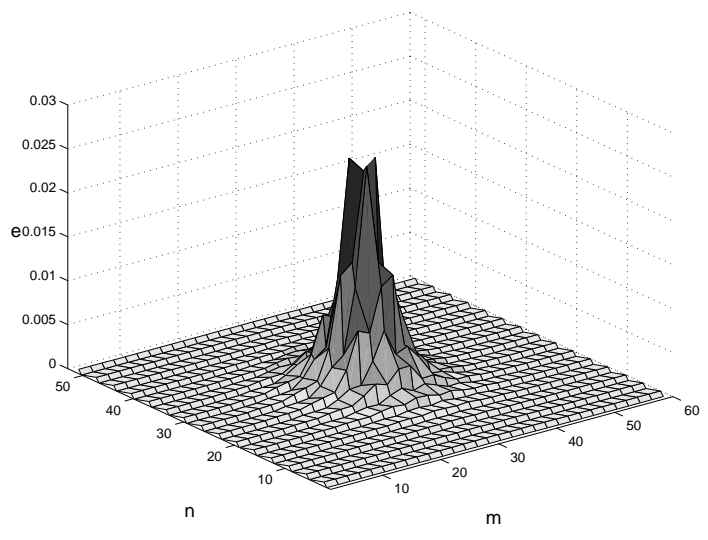

(d) Plot of $e_{m, n}, E_{0}=0.5425$.

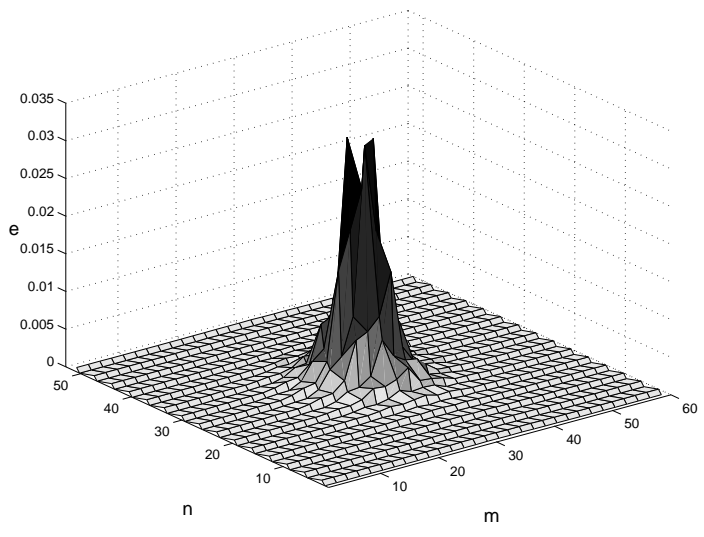

(f) Plot of $e_{m, n}, E_{0}=0.5371$.

Figure 14: Stationary breather in a lattice with an asymmetric potential, see Section 4.8 for details. 
required for stationary breathers and becomes vanishingly small at the boundary of the domain of ellipticity.

In Section 3, we extended the small amplitude expansion to fifth-order and derived a higher-order equation which more correctly describes the shape and stability properties of the breather envelope. We obtained a generalised NLS equation (3.5) with a variety of perturbation terms, some of which are known to be stabilising. This equation is slightly simpler than the corresponding equation obtained for the square lattice, namely (3.11) of [2]. In particular, the higher-order dispersive terms in (3.5) are isotropic, reflecting the hexagonal rotational symmetry of the lattice. For stationary breathers in the case of a symmetric potential we find that the cubic nonlinearity does not give rise to third harmonics, that is, $H_{3}=0$ in the hexagonal lattice, in contrast to $H_{3}=b F^{3} / 8$ in the square lattice.

In Section 4, we illustrated these breather modes, showing that both stationary and moving breathers are long-lived. The breather profiles change little over time, a small amount of energy is shed due to the initial conditions being only approximate. We have successfully propagated long-lived breathers moving in directions which are not axes of symmetry of the lattice (for instance, $\Psi=130^{\circ}$ ), suggesting that there is no absolute restriction upon the direction of travel; this is in contrast to the observations reported by Marin et al [15, 16] who could only find breathers which travelled along axes of symmetry of the lattice. There are two distinctions between these two cases: our results are for a scalar system whereas Marin et al studied a mechanical (twocomponent) two-dimensional lattice. Also, our predicted asymptotic calculations lead to small-amplitude breathers which are wide relative to the lattice spacing where it is valid to make quasi-continuum approximations; the breathers studied by Marin et al are firmly in the discrete parameter regime.

\section{Acknowledgments}

IAB would like to thank both the UK Engineering and Physical Sciences Research Council for financial assistance, and also Qamran Yaqoob for his assistance in the preparation of several diagrams that appear in this paper.

\section{Appendix A. Approximation to Townes soliton}

Since analytic formulae for Townes solitons are unavailable, we use the RayleighRitz method to find time-harmonic radially symmetric solutions of

$$
\mathrm{i} F_{T}+D \nabla^{2} F+B|F|^{2} F=0,
$$

of the form $F(\mathbf{x}, T)=e^{\mathrm{i} \lambda T} \phi(r)$ where $r=|\mathbf{x}|=\sqrt{\xi^{2}+\eta^{2}}$. The function $\phi$ satisfies

$$
-\lambda \phi+D \nabla^{2} \phi+B \phi^{3}=0,
$$

where $\nabla^{2}=\partial_{\xi}^{2}+\partial_{\eta}^{2}$. Equation (A2) arises from a variational derivative of

$$
\mathcal{E}(\phi)=\iint \frac{1}{2} \lambda|\phi|^{2}+\frac{1}{2} D|\nabla \phi|^{2}-\frac{1}{4} B|\phi|^{4} d^{2} \mathbf{r} .
$$


Using a trial solution of the form $\phi=\alpha \operatorname{sech}(\beta r)$, where $\alpha$ and $\beta$ are undetermined parameters, we find

$$
\mathcal{E}(\alpha, \beta)=\frac{D(1+2 \ln 2)}{12} \alpha^{2}-\frac{B(4 \ln 2-1)}{24} \frac{\alpha^{4}}{\beta^{2}}+\frac{\lambda \ln 2}{2} \frac{\alpha^{2}}{\beta^{2}} ;
$$

$\alpha$ and $\beta$ are determined by seeking stationary points of the action $\mathcal{E}$, namely $\partial \mathcal{E} / \partial \alpha=$ $\partial \mathcal{E} / \partial \beta=0$. Hence we find

$$
\alpha=\sqrt{\frac{12 \lambda \ln 2}{B(4 \ln 2-1)}}, \quad \beta=\sqrt{\frac{6 \lambda \ln 2}{D(2 \ln 2+1)}},
$$

and so our approximation to the Townes soliton solution of (A1) is

$$
F=\sqrt{\frac{12 \lambda \log 2}{B(4 \log 2-1)}} \exp (\mathrm{i} \lambda T) \operatorname{sech}\left(\sqrt{\frac{6 \lambda \log 2}{D(2 \log 2+1)}} \sqrt{\xi^{2}+\eta^{2}}\right) .
$$

\section{References}

[1] IA Butt. PhD Thesis. University of Nottingham, to be published at http://etheses.nottingham.ac.uk/ (2006).

[2] IA Butt and JAD Wattis. Discrete breathers in a two-dimensional Fermi-Pasta-Ulam lattice. $J$. Phys. A: Math. Gen., 39, 4955, (2006).

[3] IA Butt and JAD Wattis. Asymptotic analysis of combined breather-kink modes in a Fermi-PastaUlam chain. submitted, (2006).

[4] TA Davydova, AI Yakimenko, and YA Zaliznyak. Two-dimensional solitons and vortices in normal and anomalous dispersive media. Phys. Rev. E, 67, 026402, (2003).

[5] E Fermi, J Pasta, and S Ulam. Studies of nonlinear problems. Los Alamos Scientific Report, 1940, originally unpublished. Later published in Lectures in Applied Mathematics, 15, 143, (1974).

[6] G Fibich and G Papanicolaou. A modulation method for self-focusing in the perturbed critical nonlinear Schrödinger equation. Phys. Lett. A, 239, 167, (1998).

[7] G Fibich and G Papanicolaou. Self-focusing in the perturbed and unperturbed nonlinear Schrödinger equation in critical dimension. SIAM J. Appl. Math., 60, 183, (1999).

[8] S Flach, K Kladko, and RS MacKay. Energy thresholds for discrete breathers in one-, two-, and three-dimensional lattices. Phys. Rev. Lett., 78, 1207, (1997).

[9] S Flach, K Kladko, and CR Willis. Localized excitations in two-dimensional lattices. Phys. Rev. $E, \mathbf{5 0}, 2293$, (1994).

[10] J Giannoulis and A Mielke. The nonlinear Schrödinger equation as a macroscopic limit for an oscillator chain with cubic nonlinearities. Nonlinearity, 17, 551, (2004).

[11] J Giannoulis and A Mielke. Dispersive evolution of pulses in oscillator chains with general interaction potentials. Disc. Cont. Dyn. Sys. B, 6, 493, (2006).

[12] M Kastner. Energy thresholds for discrete breathers. Phys. Rev. Lett., 92, 104301, (2004).

[13] EA Kuznetsov, AM Rubenchik and VE Zakharov. Soliton stability in plasmas and hydrodynamics. Phys Rep, 142, 103, (1986).

[14] RS MacKay and S Aubry. Proof of existence of breathers for time-reversible or Hamiltonian networks of weakly coupled oscillators. Nonlinearity, 7, 1623, (1984).

[15] JL Marin, JC Eilbeck, and FM Russell. Localised moving breathers in a 2D hexagonal lattice. Phys. Lett. A, 248, 225, (1998).

[16] JL Marin, JC Eilbeck, and FM Russell. 2D breathers and applications (Nonlinear Science at the Dawn of the 21st Century). Eds: PL Christiansen and MP Soerensen. Springer, Berlin, (2000).

[17] JL Marin, FM Russell, , and JC Eilbeck. Breathers in cuprate-like lattices. Phys. Lett. A, 281, $21,(2001)$. 
[18] M Remoissenet. Low-amplitude breather and envelope solitons in quasi-one-dimensional physical models. Phys. Rev. B, 33, 2386, (1985).

[19] MI Weinstein. Excitation thresholds for nonlinear localized modes on lattices. Nonlinearity, 12, 673, (1999). 